\title{
On the Nisan-Ronen conjecture for submodular valuations
}

\author{
George Christodoulou* Elias Koutsoupias ${ }^{\dagger} \quad$ Annamária Kovács $^{\ddagger}$
}

\begin{abstract}
We consider incentive compatible mechanisms for a domain that is very close to the domain of scheduling $n$ unrelated machines: the single exception is that the valuation of just one machine is submodular. For the scheduling problem with such cost functions, we give a lower bound of $\Omega(\sqrt{n})$ on the approximation ratio of incentive compatible deterministic mechanisms. This is a strong information-theoretic impossibility result on the approximation ratio of mechanisms on relatively simple domains. The lower bound of the current work assumes no restriction on the mechanism side, but an expanded class of valuations, in contrast to previous general results on the Nisan-Ronen conjecture that hold for only special classes of mechanisms such as local, strongly monotone, and anonymous mechanisms. Our approach is based on a novel characterization of appropriately selected smaller instances that allows us to focus on particular type of algorithms (linear mechanisms), from which we extract a locality property that gives the lower bound.
\end{abstract}

\section{Introduction}

The design of protocols that provide appropriate incentives to participants, entice them to cooperate, and behave in a way which is socially beneficial has a long and celebrated history. It is the realm of mechanism design which is one of the most researched branches of Game Theory and Microeconomics. It studies the design of algorithms, called mechanisms, and it has numerous applications to many situations in modern societies, whenever a protocol of conduct of selfish participants is required. The mechanism asks each participant to bid their preferences over the different social outcomes, and implements one of them (e.g. the one which is most socially beneficial). The challenge is that the preferences of the participants are private, and they are either unmotivated to report correctly, or strongly motivated to report them erroneously, if a false report is profitable. A truthful mechanism provides incentives in a way that it is in the best interest of each participant to bid truthfully.

The algorithmic nature of mechanism design and the associated computational issues, were brought to light, in the seminal 20-year-old paper by Nisan and Ronen [31], which essentially established the area of algorithmic mechanism design. They proposed the scheduling problem on unrelated machines, a fundamental, extensively studied from the algorithmic perspective, optimization problem as a representative specimen to study the limitations of truthful mechanisms. The objective is to incentivize $n$ machines to execute $m$ tasks, so that the maximum completion time of the machines, i.e. the makespan, is minimized.

Nisan and Ronen applied the famous Vickrey-Clarke-Groves (VCG) mechanism [33, 12, 21 which is a general machinery that truthfully computes the outcome that maximizes the social welfare, which for the case of scheduling is the allocation that minimizes the sum of completion times. The VCG is truthful and polynomial-time for scheduling, but with respect to the makespan minimization it has a rather poor approximation ratio, equal to the number of machines $n$. Despite this, they conjectured that the VCG is the mechanism with the best approximation ratio for this problem.

\footnotetext{
${ }^{*}$ Department of Computer Science, University of Liverpool, UK. Email: gchristo@liverpool.ac.uk

${ }^{\dagger}$ Department of Computer Science, University of Oxford, UK. Email: elias@cs.ox.ac.uk

${ }^{\ddagger}$ Department of Informatics, Goethe University, Frankfurt M., Germany. Email: panni@cs.uni-frankfurt.de
} 
Conjecture 1. There is no deterministic truthful mechanism with approximation ratio better than $n$ for the problem of scheduling $n$ unrelated machines.

The bound in the conjecture is information-theoretic, in the sense that it should hold for all mechanisms (algorithms), polynomial-time or not. The Nisan-Ronen conjecture developed into one of the central problems in Algorithmic Game Theory, and despite intensive efforts only very sparse progress has been made towards its resolution. The original Nisan-Ronen paper showed that no truthful deterministic mechanism can achieve an approximation ratio better than 2 . This was improved to 2.41 [10], and later to 2.61 [23], which leaves a huge gap with the known upper bound of $n$. The most general and interesting result, by Ashlagi et al.[3], resolved a restricted version of the conjecture, for the special yet natural class of anonymous mechanisms. However, the original conjecture (for non-anonymous mechanisms) is widely open.

The original conjecture was posed for the case of additive valuations, where the total cost of each machine equals the sum of its individual costs of each task. However, many mechanism design settings consider more general valuations such as submodular, subadditive etc. In these valuations, the cost of a machine that takes a set of tasks $S$ is a function of $S$ that satisfies some natural properties. For example, for subadditive valuations the cost of a bundle of tasks $S$ can be any value bounded above by the sum of the cost of the individual tasks in $S$. This extended domain may affect significantly the incentive compatibility aspect of mechanisms because it allows a machine to also lie about the cost of bundles of tasks.

It is natural to pose the Nisan-Ronen question for these extended valuation classes. Despite the importance of the problem and various attempts, the conjecture has been widely open for all of them. In this work, we consider submodular cost functions, where the marginal contribution of a task to the total cost of a machine is a non-increasing function. The class of submodular valuations contains all additive valuations, it is a proper subject of subadditive valuations, and it is one of the most restrictive natural valuations for the scheduling question. Furthermore it is the most-studied class of valuations for the corresponding maximization problem of combinatorial auctions (see for example [25, 18, 15]).

\subsection{Our result}

We give the first non-constant lower bound that works for all deterministic truthful mechanisms when the cost functions are submodular.

Theorem 1. There is no deterministic truthful mechanism with approximation ratio better than $\sqrt{n-1}$ for the problem of scheduling $n$ unrelated machines with submodular cost functions.

Actually we show a stronger result: Let $\mathcal{M}$ be the class of scheduling mechanisms which are deterministic and truthful when all machines are additive except for one machine, which is submodular. Then no mechanism in $\mathcal{M}$ has an approximation ratio better than $\sqrt{n-1}$ on the set of instances of scheduling unrelated additive machines.

The lower bound of the current work assumes no restriction on the mechanism side, but an expanded class of valuations. In contrast, the Nisan-Ronen conjecture has been shown to hold for only special classes of mechanisms (local [31, strongly monotone [30], and anonymous [3] mechanisms).

Other valuation classes: Actually we don't anticipate any difficulty in translating, in fact carbon-copying, our results to supermodular valuations; we will include the details in the final version of the paper. Interestingly, the class of additive valuations of the original Nisan-Ronen conjecture is exactly the intersection of submodular and supermodular valuations.

In fact, we provide a stronger version of our theorem by considering submodular functions which are also $\epsilon$-additive, in the sense that the execution time of a set of tasks is within an arbitrarily small $\epsilon$ from the sum of the execution times of its tasks (see Section 2 for a precise 
definition). An important feature of our lower bound construction is that the cost functions of all machines are additive with the single exception of one machine which is submodular for particular disjoint pairs of tasks.

\subsection{Overview of the techniques}

We provide an overview of our approach for the lower bound. We consider instances in which every task has a fixed large value, practically infinite, for all except for two machines; one of the two machines is always the submodular player (player 0). We can assume that these tasks are allocated to one of the two machines, otherwise the approximation ratio is sufficiently high. This restriction of the allocation to only two players per task, have been previously used (e.g. [10, 23]). The main difference with previous approaches is that we use properties of mechanisms that involve at least three players, the submodular player and two other players. Obtaining such multiplayer statements is the bottleneck for a complete characterization of mechanisms in multiplayer domains.

Two-player characterization. We first focus on the tasks that can be allocated to a particular additive player and fix the values of the remaining tasks. For each additive player, there are only two such tasks, and the situation is very similar to two-player and two-task special case in which the valuation of one player is submodular. A core part of our proof, which is also of independent interest, is a characterization of the allocation functions of all truthful mechanisms for this case.

We provide a complete characterization for the case of two players with submodular valuations which are non-negative and are bounded above by a constant. This is essential in our construction to guarantee that the fixed large value of the other machines does not play any role. Since this is a minimization problem, it is the lower bound (i.e., the restriction that the values are non-negative) that creates complications rather then the upper bound on the domain. We also provide a characterization for additive valuations, the actual scheduling domain.

We note that similar two-player characterizations have been provided by previous work [17, 9], for auctions and scheduling domains, but none of these can be used in our approach. In particular, the characterization of [17] for scheduling relies extensively on the bounded approximation for two players, but we need a characterization without this assumption. The reason is that the approximation ratio of any two players is in general unrelated to the approximation ratio of the whole multi-player instance. In the same work, a characterization for auctions with subadditive valuations is provided, but this is also of no use to the minimization we consider here. Finally, the characterization of [9] cannot be used because it allows negative values.

Indeed, as we show in Section 3, the scheduling domain admits truthful mechanisms not present in the previous characterizations of [17, 9], which we call relaxed affine minimizers. Such mechanisms are essentially affine minimizers for large values, but they can have non-linear boundaries for small values (see Definition 5 and Figure 2).

Gluing two-player mechanisms to multi-player linear mechanisms. From the characterization of two-payer two-task mechanisms and using the fact that we are interested in mechanisms with small approximation ratio for the whole multi-player instances, we are able to exclude all mechanisms except of those that have affine boundaries (Lemma 3), that is, affine minimizers whose coefficients may depend on the values of the other tasks.

One of the main technical steps is that we use the truthfulness of the submodular player to show that the scaling coefficient of these linear mechanisms do not actually depend on the values of the other tasks (Lemma 4). The rest of the proof, which includes the most complicated technical steps of this work, is to analyze the properties of truthful linear algorithms that facilitate the proof of the lower bound (Lemma 6). 


\subsection{Related work}

The problem of scheduling unrelated machines is a typical multi-dimensional mechanism design problem. In multi-dimensional mechanism design, the valuation of each player for different outcomes is expressed via a vector (one value for every outcome). In the case of unrelated scheduling, this vector expresses the processing times of a machine for each subset of tasks and can be succinctly represented by an $m$-valued vector, one value for each task.

An interesting special case, which is well-understood, is the single-dimensional mechanism design in which the values of the vector are linear expressions of a single parameter. The principal representative is the problem of scheduling related machines, where the cost of each machine can be expressed via a single parameter, its speed. This was first studied by Archer and Tardos [2] who showed that, in contrast to the unrelated machines version, an algorithm that minimizes the makespan can be truthfully implemented - albeit in exponential time. It was subsequently shown that truthfulness has essentially no impact on the computational complexity of the problem. Specifically, a randomized truthful-in-expectation ${ }^{1}$ PTAS was given in [14] and a deterministic PTAS was given in [11]; a PTAS is the best possible algorithm even for the pure algorithmic problem (unless $P=N P$ ).

The main obstacle in resolving the Nisan-Ronen conjecture is the lack of clear algorithmic understanding of truthfulness for many players in multi-dimensional domains. In contrast, we understand better truthfulness for a single player. Saks and Yu 32 gave a nice, complete characterization of deterministic truthful mechanisms for convex domains which was later extended to truthful-in-expectation randomized mechanisms in [1]. This characterization states that the class of allocations of truthful mechanisms is the class of weakly monotone algorithms [5]. This is an elegant characterization, but it has not been proved very useful for mechanisms of many players, because it is difficult to combine monotonicity of each individual player into a single global condition. Its direct applications have provided only constant bounds for makespan minimization [10, 30, 23, 31]. What would be more useful is a characterization similar to the one provided by the seminal work of Roberts [22] for unrestricted domains. It essentially states that the only truthful mechanisms are affine extensions of VCG. Similar characterizations have been provided in [9, 17, 16] for settings with only two players. Extending these characterizations to multiple players for scheduling and combinatorial auctions is notoriously hard, mainly due to lack of externalities in these settings: the valuation of a player for an allocation depends only on the subset of tasks it receives and is indifferent on how the remaining of the tasks are assigned to the other player: 2 .

Scheduling is related to combinatorial auctions, where multiple items need to be assigned to a set of buyers. This is a broad and successful area, and the setting shares both aforementioned features of multi-dimensionality and lack of externalities, therefore insights and techniques can be transferred from the one problem to the other. However the difference is that the objective for combinatorial auctions is social welfare maximization, and this is known to be achieved by the VCG mechanism, albeit in exponential time. Hence the focus on this rich area is on what can be achieved by computationally efficient mechanisms (see for example [18]). But in the case of the scheduling with the min-max objective, the flavor is more information theoretic, as we know that not even exponential time mechanisms can achieve the optimal makespan.

\subsubsection{Further related work}

Lavi and Swamy [24] proposed an interesting approach to attack the Nisan-Ronen question, by restricting the input domain, but still keep the multi-dimensional flavour of the setting. They

\footnotetext{
${ }^{1}$ This is one of the two main definitions of truthfulness for randomized mechanisms, where truth-telling maximizes the expected utility of each player.

${ }^{2}$ For two players, there exist implicit externalities as the tasks one player doesn't get determine what the other player gets.
} 
assumed that each entry in the input matrix can take only two possible values "low" and "high", that are publicly known to the designer. In this case, they showed an elegant deterministic mechanism with an approximation factor of 2. Surprisingly, even for this special case there is a lower bound of 11/10. Yu [34] extended the results for a range of values, and Auletta et al. [4] studied multi-dimensional domains where the private information of the machines is a single bit.

Randomization has been explored and slightly improved the known guarantees. There are two notions of truthfulness for randomized mechanisms. A mechanism is universally truthful if it is defined as a probability distribution over deterministic truthful mechanisms, while it is truthful-in-expectation, if in expectation no player can benefit by lying. In [31, a universally truthful mechanism was proposed for the case of two machines, and was later extended to the case of $n$ machines by Mu'alem and Schapira [30] with an approximation guarantee of $0.875 n$, which was later improved to $0.837 n$ by [28]. Lu and $\mathrm{Yu}$ [29] showed a truthful-in-expectation mechanism with an approximation guarantee of $(m+5) / 2$. Mu'alem and Schapira [30, showed a lower bound of $2-1 / m$, for both notions of randomization. Christodoulou, Koutsoupias and Kovács [8] extended the lower bound for fractional mechanisms, where each task can be fractionally allocated to multiple machines. They also showed a fractional mechanism with a guarantee of $(m+1) / 2$. A sequence of papers studied randomized mechanisms for the special case of two machines [27, 29] where a tight answer on the approximation factor is still unresolved. Currently, the best upper bound is 1.587 due to Chen, Du, and Zuluaga [7].

The truthful implementation of other objectives have been explored by Mu'alem and Schapira [30] for multi-dimensional problems and by Epstein and van Stee [19] for single-dimensional ones, giving a PTAS for a wide range of objective functions. Leucci, Mamageishvili and Penna [26] showed high lower bounds for other min-max objectives on some combinatorial optimization problems. In the Bayesian setting, Daskalakis and Weinberg [13] showed a mechanism that is at most a factor of 2 from the optimal truthful mechanism, but not with respect to optimal makespan. Chawla et al. [6] provided bounds of prior-independent mechanisms (where the input comes from a probability unknown to the mechanism). Giannakopoulos and Kyropoulou [20] showed that the VCG mechanism achieves an approximation ratio of $O(\log n / \log \log n)$ under some distributional and symmetry assumptions.

\section{Preliminaries}

There is a set $N$ of $n$ machines and a set $M$ of $m$ tasks that need to be scheduled on the machines. The processing time or cost that each machine $i$ takes to process a subset $S$ of tasks is described by a set function $t_{i}: 2^{m} \rightarrow \mathbb{R}_{\geq 0}$. In classic unrelated machines scheduling, the cost functions are additive and the objective is to minimize the makespan (min-max objective).

We also consider more general cost functions that are normalized $\left(t_{i}(\emptyset)=0\right)$ and monotone $\left(t_{i}(S) \leq t_{i}(T)\right.$ for $\left.S \subseteq T\right)$. We focus on submodular cost functions, which satisfy the following condition for every $S, T \subseteq M$

$$
t_{i}(S \cup T)+t_{i}(S \cap T) \leq t_{i}(S)+t_{i}(T) .
$$

In the classical Nisan-Ronen model, the cost functions are additive. In our lower bound construction, we will assume that all cost functions are additive except for one which is submodular. We also consider valuations which are arbitrarily close to additive, which we call $\epsilon$-additive, such that for every subset $S, \sum_{j \in S}\left(t_{i}(\{j\})\right)-\epsilon \leq t_{i}(S) \leq \sum_{j \in S}\left(t_{i}(\{j\})\right)+\epsilon$. Our results hold for valuations that are both submodular and $\epsilon$-additive - even for valuations within an $\epsilon$ multiplicative factor from additive.

Mechanism design setting. We assume that each machine $i \in N$ is controlled by a selfish agent that is reluctant to process the tasks and the cost function $t_{i}$ is private information known 
only to her (also called the type of agent $i$ ). In the most general version of the problem, the set $\mathcal{T}_{i}$ of possible types of agent $i$ consists of all vectors $b_{i} \in \mathbb{R}_{+}^{2^{m}}$. Let also $\mathcal{T}=\times_{i \in N} \mathcal{T}_{i}$ be the space of type profiles.

A mechanism defines for each player $i$ a set $\mathcal{B}_{i}$ of available strategies, the player (agent) can choose from. We will consider direct revelation mechanisms, i.e., $\mathcal{B}_{i}=\mathcal{T}_{i}$ for all $i$, meaning that the players strategies are to simply report their types to the mechanism. A player may report a false cost function $b_{i} \neq t_{i}$, if this serves her interests.

A mechanism $(A, P)$ consists of two parts:

An allocation algorithm: The allocation algorithm $A$ allocates the jobs to the machines depending on the players' bids $b=\left(b_{1}, \ldots, b_{n}\right)$. Let $\mathcal{A}$ be the set of all possible partitions of $m$ tasks to $n$ machines. The allocation function $A: \mathcal{T} \rightarrow \mathcal{A}$ partitions the tasks into the $n$ machines; we denote by $A_{i}(b)$ the subset of tasks assigned to machine $i$ in the bid profile $b$.

A payment scheme: The payment scheme $P=\left(P_{1}, \ldots, P_{n}\right)$ determines the payments also depending on the bid values $b$. The functions $P_{1}, \ldots, P_{n}$ stand for the payments that the mechanism hands to each agent i.e. $P_{i}: \mathcal{T} \rightarrow \mathbb{R}$.

The utility $u_{i}$ of a player $i$ is the payment that he gets minus the actual time that he needs to process the set of tasks assigned to her, $u_{i}(b)=P_{i}(b)-t_{i}\left(A_{i}(b)\right)$. We are interested in truthful mechanisms. A mechanism is truthful, if for every player, reporting his true type is a dominant strategy. Formally,

$$
u_{i}\left(t_{i}, b_{-i}\right) \geq u_{i}\left(t_{i}^{\prime}, b_{-i}\right), \quad \forall i \in[n], \quad t_{i}, t_{i}^{\prime} \in \mathcal{T}_{i}, \quad b_{-i} \in \mathcal{T}_{-i},
$$

where $\mathcal{T}_{-i}$ denotes the possible types of all players disregarding $i$.

We use as an objective to evaluate the performance of a mechanism's allocation algorithm the makespan, that is the maximum completion time of a machine. The makespan of the allocation algorithm $A$ with respect to a given input $t$ is

$$
\operatorname{Mech}(t) \stackrel{\text { def }}{=} \max _{i \in N} t_{i}\left(A_{i}(t)\right)
$$

We aim at minimizing the makespan, hence the optimum is

$$
\operatorname{Opt}(t)=\min _{A \in \mathcal{A}} \max _{i \in N} t_{i}\left(A_{i}\right)
$$

We are interested in the approximation ratio of the mechanism's allocation algorithm. A mechanism $M$ is $c$-approximate, if the allocation algorithm is $c$-approximate, that is, if $c \geq \frac{M e c h(t)}{O p t(t)}$ for all possible inputs $t$.

We are looking for truthful mechanisms with low approximation ratio irrespective of the running time to compute $A$ and $P$. In other words, our lower bounds do not make use of any computational assumptions.

Weak monotonicity. A useful characterization of truthful mechanisms in terms of the following monotonicity condition, helps us to get rid of the payments and focus on the properties of the allocation algorithm.

Definition 1. An allocation algorithm $A$ is called weakly monotone (WMON) if it satisfies the following property: for every two inputs $t=\left(t_{i}, t_{-i}\right)$ and $t^{\prime}=\left(t_{i}^{\prime}, t_{-i}\right)$, the associated allocations $A$ and $A^{\prime}$ satisfy

$$
t_{i}\left(A_{i}\right)-t_{i}\left(A_{i}^{\prime}\right) \leq t_{i}^{\prime}\left(A_{i}\right)-t_{i}^{\prime}\left(A_{i}^{\prime}\right)
$$


It is well known that the allocation function of every truthful mechanism is WMON [5], and also that this is a sufficient condition for truthfulness in convex domains [32].

A useful tool in our proof relies on the following immediate consequence of WMON, which holds in additive domains as well as in the other domains that we consider. Intuitively, it states that when you fix the values of all players for a subset of tasks (focus on a cut of your domain), then the restriction of the allocation to the rest of the tasks must remain weakly monotone.

Lemma 1. Let $A$ be a WMON allocation. Let us fix an $(S, T)$ partition of $M$, and consider only valuations $t_{i}$ of player $i$ that are additive across $S$ and $T$, i.e., for every $X \subseteq M, t_{i}(X)=$ $t_{i}(X \cap S)+t_{i}(X \cap T)$. Then the restriction of the allocation $A$ on $S$ is weakly monotone for each valuation fixed on the subsets of $T$.

Proof. $A$ is weakly monotone, therefore

$$
t_{i}\left(A_{i}\right)-t_{i}^{\prime}\left(A_{i}\right) \leq t_{i}\left(A_{i}^{\prime}\right)-t_{i}^{\prime}\left(A_{i}^{\prime}\right),
$$

and additivity across $S$ and $T$ implies

$$
t_{i}\left(A_{i} \cap S\right)-t_{i}^{\prime}\left(A_{i} \cap S\right) \leq t_{i}\left(A_{i}^{\prime} \cap S\right)-t_{i}^{\prime}\left(A_{i}^{\prime} \cap S\right),
$$

as the values of subsets of $T$ are fixed, $t_{i}\left(A_{i} \cap T\right)=t_{i}^{\prime}\left(A_{i} \cap T\right)$ and $t_{i}\left(A^{\prime}{ }_{i} \cap T\right)=t_{i}^{\prime}\left(A^{\prime}{ }_{i} \cap T\right)$.

The following lemma was essentially shown in [31] and has been a useful tool to show lower bounds for truthful mechanisms for several variants (see for example [10, 30, 3]). Although this holds more generally, we only state it (and use it in Section 4) for additive valuations.

Lemma 2. Let $t$ be a bid vector of additive valuations, and let $S=A_{i}(t)$ be the subset assigned to player $i$ by the mechanism. For any bid vector $t^{\prime}=\left(t_{i}^{\prime}, t_{-i}\right)$ such that only the bid of machine $i$ has changed and in such a way that for every task in $S$ it has decreased (i.e., $t_{i}^{\prime}(\{j\})<$ $t_{i}(\{j\}), j \in S$ ) and for every other task it has increased (i.e., $\left.t_{i}^{\prime}(\{j\})>t_{i}(\{j\}), j \in M \backslash S\right)$. Then the mechanism does not change the allocation to machine $i$, i.e., $A_{i}\left(t^{\prime}\right)=A_{i}(t)=S$.

The main challenge in multi-player settings is that the allocation of the other machines may change and the above condition makes no promise about how this can happen.

\section{Characterization for two players}

A core element of our lower bound proof is a characterization of truthful mechanisms for two tasks and two players (called $t$-player, and $s$-player). We first provide a characterization for additive valuations $t=\left(t_{1}, t_{2}\right)$, and $s=\left(s_{1}, s_{2}\right)$, so that both $s_{1}$ and $s_{2}$ are bounded by an arbitrarily large but fixed value $B$. Then we extend it when the $t$ player has submodular valuations $t=\left(t_{1}, t_{2}, t_{12}\right)$ (Theorem 2), which is the main element that we need in the lower bound in Section 4. We note that both the characterization and the lower bound result hold analogously when the $t$-player has arbitrary monotone, or $\epsilon$-additive (or submodular and $\epsilon$ additive) valuations.

We postpone the details of the characterization proof to the Appendix, but in this section we introduce the definitions that we will need in order to state the main result and also to introduce some notation which will be used in our lower bound proof in Section 4 . We keep in the Appendix intact the whole proof of the characterization. 


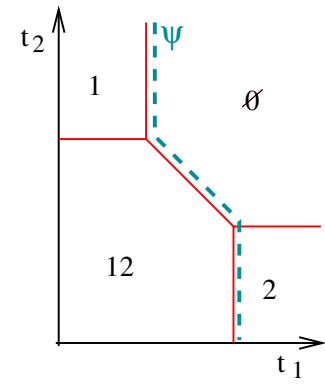

(a)

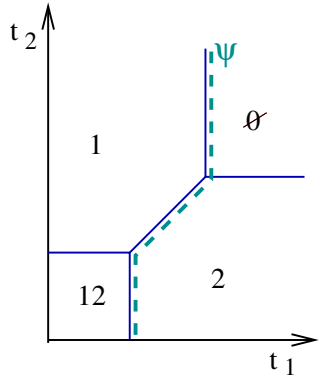

(b)

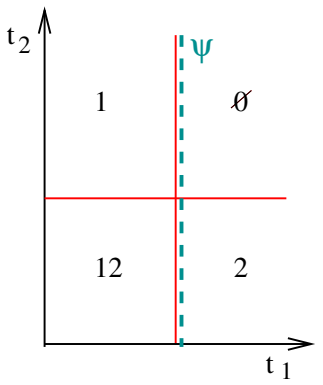

(c)

Figure 1: The allocation $A[s]$ to the $t$-player depending on his own bid vector $\left(t_{1}, t_{2}\right)$ : (a) quasi-bundling allocation; (b) quasi-flipping allocation; (c) crossing allocation. The interiors of some regions might be empty, but $R_{\emptyset} \neq \emptyset$ can be assumed w.l.o.g. We marked the functions of critical values $\psi\left(t_{2}, s\right)$ (Definition 7 ) for receiving task 1 by broken lines.

\subsection{Basic Definitions}

Let $(A, P)$ be a truthful mechanism, where $A$ is the WMON allocation function, and $P$ denotes the payment function. For input $(t, s)$ the allocation is $A(t, s)$. Since we have only tasks 1 and 2 , in $A(t, s)$ we can denote the allocation to one of the players as $\alpha_{t}, \alpha_{s} \in\{12,1,2, \emptyset\}$.

For given $s \in[0, B) \times[0, B)$ the allocation for the $t$-player as function of his own bids $\left(t_{1}, t_{2}\right)$ (or $\left(t_{1}, t_{2}, t_{12}\right)$ ) is denoted by $A[s]$, and symmetrically $A[t]$ is an allocation function for the $s$-player. For $\alpha_{t} \in\{12,1,2, \emptyset\}$, the allocation regions $R_{\alpha_{t}}(s) \subseteq \mathbb{R}_{\geq 0}^{2}$ (resp. $\mathbb{R}_{\geq 0}^{3}$ ) of $A[s]$ are defined to be the interior (wrt. $\mathbb{R}_{\geq 0}^{2}$ ) of the set of all $t$ values such that $A(t, s)=\alpha_{t}$. For the $s$-player we denote the respective regions by $R_{\alpha_{s}}^{s}(t)$.

Additive players It is known that in the case of two tasks, the regions in a WMON allocation subdivide $R_{>0}^{2}$ basically in three possible forms, which are characteristic for the type of the whole allocation-function $A$ (see Figure 1). The regions and their boundaries determine the critical values for $t_{1}$ (as function of $t_{2}$ ) above which the $t$-player cannot get task 1 , and symmetrically for task 2. These critical value functions are determined by the payment functions $P_{\emptyset}(s)=$ $0, P_{1}(s), P_{2}(s), P_{12}(s)$ for the fixed $s$.

Definition 2. For given $s$, we call the allocation $A[s]$

- quasi-bundling, if there are at least two points $t \neq t^{\prime}$ on the boundary of $R_{12}$ and $R_{\emptyset}$

- quasi-flipping, if there are at least two points $t \neq t^{\prime}$ on the boundary of $R_{1}$ and $R_{2}$

- crossing otherwise (see Figure 1).

\subsection{Mechanisms for submodular players}

We now introduce the types of WMON allocations that can occur when the $t$-player is submodular and the $s$-player is additive and bounded above by the value $B$. We denote these particular domains $\mathcal{T}_{t} \times \mathcal{T}_{s}$ for two players and two tasks by $V_{\text {submod }} \times V_{+, B}$, and refer the reader to the appendix Section $\mathrm{A}$ for detailed definitions, and also for extension of our results to other domains. We assume that there always exist high enough $t$ values so that the $t$-player receives no task, i.e., that $R_{\emptyset}(s) \neq \emptyset$ for every $s:^{3}$

\footnotetext{
${ }^{3}$ This assumption is without loss of generality for mechanisms with finite approximation of the makespan, even if some 2D cut mechanism of a WMON mechanism with more tasks and/or players is considered.
} 
1-dimensional mechanisms In a one-dimensional mechanism at most two possible allocations are ever realized. Due to the assumption on $R_{\emptyset}$, one of these must be the allocation $\emptyset$ to the $t$-player. If the two occuring allocations (for the $t$-player) are $\emptyset$ and 12 , we call the mechanism bundling mechanism. The other cases when the allocations to the $t$-player are $\emptyset$ and 1 (or $\emptyset$ and 2) are degenerate task-independent allocations, and can be defined similarly to bundling mechanisms.

Definition 3. In a bundling mechanism only the allocations $\emptyset$ and 12 can occur. There is an arbitrary, increasing function $\xi:[0, B) \rightarrow[0, \infty)$ so that if $t_{12}>\xi\left(s_{1}+s_{2}\right)$ then the $t$-player gets $\emptyset$, and if $t_{12}<\xi\left(s_{1}+s_{2}\right)$ then the $t$-player gets 12 .

If $\xi$ has a jump discontinuity in some point $s_{1}+s_{2}$ then the critical value may depend on the concrete $\left(s_{1}, s_{2}\right)$ with the given fixed sum, as long as it is between $\xi\left(\left(s_{1}+s_{2}\right)^{-}\right)$and $\xi\left(\left(s_{1}+s_{2}\right)^{+}\right)$.

\section{Relaxed affine minimizers}

Definition 4. An allocation $A$ is an affine minimizer, if there exist positive constants per player $\mu_{t}$ and $\mu_{s}$, and constants $\gamma_{\alpha} \in \mathbb{R} \cup\{-\infty, \infty\}$ per allocation (say, here $\alpha=\alpha_{t}$ ), so that for every input $(t, s)$ the allocation $A(t, s)$ minimizes over

$$
\mu_{t} t_{12}+\gamma_{12}, \quad \mu_{t} t_{1}+\mu_{s} s_{2}+\gamma_{1}, \quad \mu_{s} s_{1}+\mu_{t} t_{2}+\gamma_{2}, \quad \mu_{s}\left(s_{1}+s_{2}\right)+\gamma_{\emptyset} .
$$

Definition 5. An allocation $A$ is a relaxed affine minimizer, if there exist positive constants per player $\mu_{t}$ and $\mu_{s}$, and constants $\gamma_{\alpha}$ per allocation $\alpha$ (of the $t$-player), furthermore an arbitrary increasing function $\xi:\left[0, \min \left(\gamma_{1}, \gamma_{2}\right)-\gamma_{\emptyset}\right) \rightarrow[0, \infty)$ (if the interval $\left[0, \min \left(\gamma_{1}, \gamma_{2}\right)-\gamma_{\emptyset}\right)$ is nonempty) with $\min \left(\gamma_{1}, \gamma_{2}\right)-\gamma_{12}=\xi\left(\min \left(\gamma_{1}, \gamma_{2}\right)-\gamma_{\emptyset}\right)$, so that for every input $(t, s)$

a) if $\mu_{s} \cdot\left(s_{1}+s_{2}\right) \geq \min \left(\gamma_{1}, \gamma_{2}\right)-\gamma_{\emptyset}$, the allocation $A(t, s)$ is that of an affine minimizer with the given constants

b) if $\mu_{s} \cdot\left(s_{1}+s_{2}\right) \leq \min \left(\gamma_{1}, \gamma_{2}\right)-\gamma_{\emptyset}$, then if $\mu_{t} \cdot t_{12}>\xi\left(\mu_{s}\left(s_{1}+s_{2}\right)\right)$ then the allocation for the $t$-player is $\emptyset$ and if $\mu_{t} \cdot t_{12}<\xi\left(\mu_{s} \cdot\left(s_{1}+s_{2}\right)\right)$ then it is 12 .

The boundary conditions in the above definition are such that the affine minimizer part (a) fits with the bundling mechanism part (b) and the resulting mechanism is truthful. See Figure 2, for an example of such a mechanism in the additive domain.

Constant mechanisms In a constant mechanism the allocation is independent of the bids of at least one of the players. This property can also be interpreted as being an affine minimizer with multiplicative constant $\mu=0$. Due to the assumption on $R_{\emptyset}$ we only need to consider constant mechanisms that are independent (at least) of the $s$-player.

\subsection{The main characterization result}

The characterization that we use for the lower bound is captured by the following theorem, whose proof can be found in the Appendix.

Theorem 2. Every WMON allocation for two tasks and two players with bids $t \in V_{\text {submod }}$ and $s \in V_{+, B}$, where both tasks are always allocated, and $R_{\emptyset}(s) \neq \emptyset$ for every $s$, is one of these three types: (1) relaxed affine minimizer, (2) one-dimensional mechanism, or (3) constant mechanism.

In the Appendix we show that the same characterization (and hence our lower bound) holds for WMON allocations for various domains of valuations of the $t$-player and in particular for submodular valuations which are arbitrarily close to additive, which we denote by $V_{\epsilon} \cap V_{\text {submod }}$.

\footnotetext{
${ }^{4}$ Throughout the paper, by 'increasing' we mean 'non-decreasing'; otherwise we say 'strictly increasing'.
} 


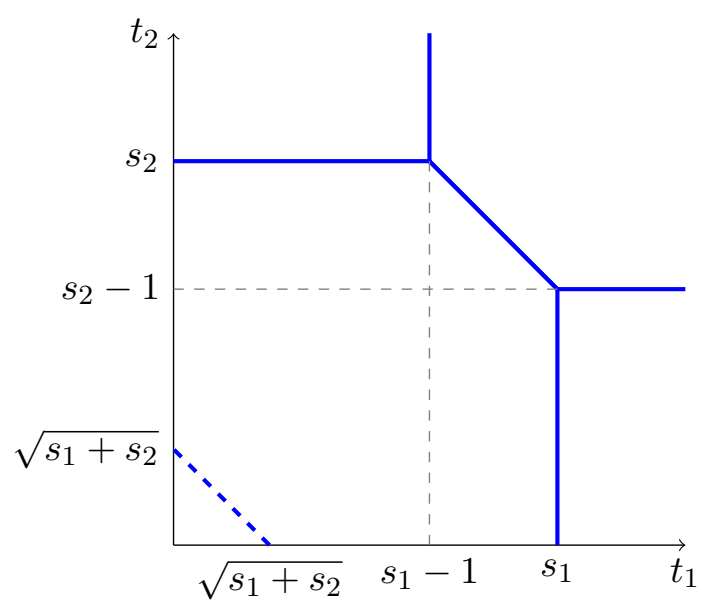

Figure 2: An example of a relaxed affine minimizer, which shows the allocation of the $t$-player. The solid lines show the boundaries of the allocations for values of the $s$-player when $s_{1}+s_{2} \geq 1$. The dashed lines show the allocation boundary when $s_{1}+s_{2}<1$. Sometimes we refer to this part of relaxed affine minimizers as "bundling tail".

\section{Lower Bound}

In this section, we give a proof of our main theorem (Theorem 1). First in Section 4.1 we describe the domain of instances that we use, and in Section 4.2 we use the characterization for two machines and two tasks (Theorem 2) to establish that the only interesting mechanisms are linear (see the subsection for a precise definition), then in Section 4.3 we explore the linearity property of mechanisms with bounded approximation ratio to establish some useful locality lemmas that are eventually used in Section 4.4 to complete the proof.

\subsection{The construction}

To prove the lower bound, we focus on the domain of $2(n-1)$ tasks and $n$ players. Player 0 is special and for convenience we use the symbol $t$ for its values; sometimes we refer to it as the $t$-player. We use the symbol $s$ for the values of the remaining players $1, \ldots, n-1$, and sometimes we refer to them as the $s$-players.

The set of tasks $M=\left\{1,1^{\prime}, 2,2^{\prime}, \ldots, n-1,(n-1)^{\prime}\right\}$ is partitioned in pairs and each pair $\left\{i, i^{\prime}\right\}$ is associated with player $i, i=1, \ldots, n-1$. We call the two tasks of each pair $\left\{i, i^{\prime}\right\}$ twin tasks.

Let $v_{i}(S)$ denote the cost (valuation) of player $i$ when it takes the subset $S \subseteq M$ of tasks.

- the cost of the $s$-players is additive: $v_{i}(S)$ is additive for $i \geq 1$.

- the cost of the $s$-players for tasks not in their associated pair is a sufficiently large fixed constant $\Theta \gg 4 n^{2}$ : for distinct $i, j \geq 1, v_{i}(\{j\})=v_{i}\left(\left\{j^{\prime}\right\}\right)=\Theta$.

- the cost of the $t$-player for twin tasks is submodular: for every $i=1, \ldots, n-1$, the restriction of $v_{0}(S)$ to $\left.S \subseteq\left\{i, i^{\prime}\right\}\right)$ is submodular.

- the cost of the $t$-player is additive across pairs: $v_{0}(S)=\sum_{i=1}^{n-1} v_{0}\left(S \cap\left\{i, i^{\prime}\right\}\right)$. Therefore $v_{0}(S)$ is submodular, as the sum of submodular functions.

To simplify the notation we will denote an instance that satisfies the above conditions by

$$
\left(t_{i}, s_{i}, t_{i^{\prime}}, s_{i^{\prime}}, t_{i, i^{\prime}}\right)_{i=1}^{n-1},
$$

where 
- $s_{i}$ and $s_{i^{\prime}}$ the cost of $i$-th $s$-player for the tasks in its associated pair $\left\{i, i^{\prime}\right\}$

- $t_{i}$ and $t_{i^{\prime}}$ the cost of the $t$-player when it takes only one of the twin tasks $\left\{i, i^{\prime}\right\}$

- $t_{i, i^{\prime}}$ the cost of the $t$-player when it takes both twin tasks $\left\{i, i^{\prime}\right\}$

With the exception of costs $t_{i, i^{\prime}}$ an instance is captured by the following matrix indexed by players and tasks, which shows the cost of each when a player gets no other task.

$$
\left[\begin{array}{ccccccc}
t_{1} & t_{1^{\prime}} & t_{2} & t_{2^{\prime}} & \cdots & t_{n-1} & t_{(n-1)^{\prime}} \\
s_{1} & s_{1^{\prime}} & \Theta & \Theta & \cdots & \Theta & \Theta \\
\Theta & \Theta & s_{2} & s_{2^{\prime}} & \cdots & \Theta & \Theta \\
& & & \vdots & & & \\
\Theta & \Theta & \Theta & \Theta & \cdots & s_{n-1} & s_{(n-1)^{\prime}}
\end{array}\right]
$$

If the valuations of all players were additive, this matrix would be sufficient to determine the cost for all bundles. The instances we consider have more general cost functions and include the submodular valuation of twin tasks for the $t$-player. Since for two tasks, a function is submodular if and only if it is subadditive, values $t_{i, i^{\prime}}$ satisfy

$$
t_{i, i^{\prime}} \leq t_{i}+t_{i^{\prime}}
$$

It is useful to think of the value $\Theta$ as practically infinite, since it is much larger that the other values. On the other hand, to prove our main theorem in its generality, we need this value to be finite and this complicates the characterization of truthful mechanisms.

We focus on instances that satisfy $s_{i} \in[0,1], s_{i^{\prime}}=n, t_{i^{\prime}}=0, t_{i, i^{\prime}}=t_{i}+t_{i^{\prime}}$ for all $i \in[n-1]$. Note the subtlety here: while the domain contains instances with $t_{i, i^{\prime}} \leq t_{i}+t_{i^{\prime}}$, for the proof of the lower bound, we consider the subclass of additive instances. This should not be surprising in the sense that lower bound proofs usually employ a subclass of instances. Still, it raises the question whether we could carry out the same proof in the additive domain. The answer is negative, because the sets of mechanisms for additive and submodular (subadditive) domains for two tasks are different; for example, task-independent mechanisms are not truthful for submodular domains.

Definition 6 (Restricted $(t, s)$ instance). Instances of the form $\left(t_{i}, s_{i}, t_{i^{\prime}}, s_{i^{\prime}}, t_{i, i^{\prime}}\right)_{i=1}^{n-1}$ (Equation 1) that satisfy

$$
\begin{aligned}
& s_{i} \in[0,1], \quad s_{i^{\prime}}=n, \quad t_{i^{\prime}}=0, \quad t_{i, i^{\prime}}=t_{i}+t_{i^{\prime}}, \quad \text { for all } i \in[n-1], \\
& \quad(t, s)=\left(\left(t_{i}\right)_{i=1}^{n-1},\left(s_{i}\right)_{i=1}^{n-1}\right)
\end{aligned}
$$

will be called restricted $(t, s)$ instances.

Note that, unlike the other values, the $t_{i}$ values can be arbitrarily high. This will be useful later (Lemma 3). Note also that the optimum makespan of every restricted instance is at most 1. For these instances, any algorithm with approximation ratio less than $n$, must allocate all the $i^{\prime}$ tasks to the $t$-player and every $i$ task either to the associated $s_{i}$-player or the $t$-player.

\subsection{From affine minimizers for twin tasks to linear mechanisms}

In this section, we use the characterization of mechanisms (Theorem 2 ) for each pair of twin tasks in an instance $\left(t_{i}, s_{i}, t_{i^{\prime}}, s_{i^{\prime}}, t_{i, i^{\prime}}\right)_{i=1}^{n-1}$, to derive an essential property of mechanisms for the restricted $(t, s)$ instances. By the characterization, there are mechanisms of twin tasks, such as the one-dimensional, constant, or bundling tails of relaxed affine minimizers, that may have non-affine boundaries between different allocations. In this section, we show that mechanisms with non-affine boundaries are also excluded, when we additionally require that the mechanisms have small approximation ratio - on whole instances, not just on a pair of twin tasks.

First we define the notion of boundaries that we consider in proof of the lower bound. 
Definition 7. For a given allocation algorithm, considered on restricted $(t, s)$ instances, we call $\psi_{i}\left(s_{i}, s_{-i}, t_{-i}\right)$ a critical value or boundary if the $t$-player receives task $i$ when $t_{i}<\psi_{i}\left(s_{i}, s_{-i}, t_{-i}\right)$, and does not receive task $i$ when $t_{i}>\psi_{i}\left(s_{i}, s_{-i}, t_{-i}\right.$ ) (for example in Figure 1, $\psi_{1}$ is shown with dashed lines).

We claim below that a truthful allocation with a reasonable approximation ratio (say, of at most $n$ ) for the restricted $(t, s)$ instances satisfies the following important property:

Definition 8 (Linear mechanisms). An allocation algorithm for the restricted $(t, s)$ instances (Equation 2), is called linear if the critical values for task $i, i=1, \ldots, n-1$ are truncated affine functions in $s_{i}$. In particular when

$$
\psi_{i}\left(s_{i}, s_{-i}, t_{-i}\right)=\max \left(0, \lambda_{i}\left(s_{-i}, t_{-i}\right) s_{i}+\gamma_{i}\left(s_{-i}, t_{-i}\right)\right),
$$

for some $\lambda_{i}\left(s_{-i}, t_{-i}\right)>0$. We call a mechanism linear, if it uses a linear allocation algorithm.

Lemma 3. A mechanism for the instances $\left(t_{i}, s_{i}, t_{i^{\prime}}, s_{i^{\prime}}, t_{i, i^{\prime}}\right)_{i=1}^{n-1}$ (Equation 1) with approximation ratio less than $n$ must be linear for the restricted $(t, s)$ instances.

Proof. By Lemma 1, the restriction of the mechanism for the twin tasks $i, i^{\prime}$ is weakly monotone, and therefore by the characterization theorem (Theorem 2), it is either a relaxed affine minimizer, a one-dimensional, or a constant mechanism. We will show that if the approximation ratio is less than $n$, the mechanism cannot be one-dimensional, constant, or even relaxed affine minimizer with a bundling tail. The reason is that algorithms in all these cases are so inefficient that their makespan only for these two tasks is large enough to show a large approximation ratio for the entire instance.

Let's first argue that it cannot be a one-dimensional or a constant mechanism. We fix all values of the other tasks as in restricted instances. By Lemma B.3.6, there are values for the twin tasks $i, i^{\prime}$ for which the algorithm has makespan $\mu \geq \sqrt{\Theta}$ and approximation ratio at least $\sqrt{\Theta}$. Since the optimal makespan of the other tasks of a restricted instance is at most 1 , the approximation ratio is at least $\mu /(1+\mu / \sqrt{\Theta}) \geq \sqrt{\Theta} / 2 \geq n$.

As a result, the restriction to twin tasks $i, i^{\prime}$ is a relaxed affine minimizer. We now argue that there should be no bundling part of the relaxed affine minimizer when the approximation ratio is less than $n$. Let us fix the values $s_{j} \in[0,1], s_{j^{\prime}}=n$ for all $j \in[n-1]$, and the values $t_{j}^{\prime}=0, t_{j, j^{\prime}}=t_{j}+t_{j^{\prime}}$ for all $j \neq i$. We observe that the restriction of the mechanism in the cut $t_{-\left\{i, i^{\prime}\right\}}$ (the 2-dimensional partition when we also fix all other values except $t_{i}$ and $t_{i}^{\prime}$ ) should definitely contain an area where the $t$-player gets task $i^{\prime}$ but not $i$ (in particular, when $t_{i^{\prime}}=\delta$, for some arbitrarily small $\delta$, and $t_{i}$ is very large). Otherwise the mechanism has approximation ratio at least $n$.

This shows that in the domain of restricted $(t, s)$ instances, the mechanisms with approximation ratio less than $n$ are affine minimizers in every cut of pairs of twin tasks (even if in general, that is, for $s_{i^{\prime}}<n$ they are relaxed affine minimizers). Therefore, the only mechanisms with approximation ratio less than $n$ are linear for the restricted $(s, t)$ instances.

\subsection{Linearity}

From now on we focus on linear truthful mechanisms for restricted $(t, s)$ instances. Establishing linearity of the boundaries (Lemma 3) is not directly useful, because the linear coefficient of the boundary $i$ may depend on the other values of $t$. The next lemma shows that this is not the case for the scaling factor $\lambda_{i}$, although the $\gamma_{i}$ term may still depend on the other values of $t$. Its proof is based on the interaction of pairs of tasks $i$ and $j$. Note that these are not twin tasks but involve at least three players, the $t$-player and the two associated $s$-players. Obtaining such multiplayer statements is the bottleneck for a complete characterization of mechanisms in multiplayer domains, and it is perhaps the most crucial part of the proof. 
Lemma 4. For fixed $i$ and $j$, and for fixed $s_{-i}, t_{-i j}$, assume that $\psi_{i}\left(s_{i}, s_{-i}, t_{-i}\right)$ is a truncated linear function of $s_{i}$, i.e., $\psi_{i}\left(s_{i}, s_{-i}, t_{-i}\right)=\max \left(0, \lambda_{i}\left(s_{-i}, t_{-i}\right) s_{i}+\gamma_{i}\left(s_{-i}, t_{-i}\right)\right)$, for some $\lambda_{i}\left(s_{-i}, t_{-i}\right) \geq 0$. Then $\lambda_{i}\left(s_{-i}, t_{-i}\right)$ is the same for all $t_{j}$ that satisfy $\psi_{i}\left(s_{i}, s_{-i}, t_{-i}\right)>0$.

Proof. To keep the notation simple, we drop all values except for $s_{i}$ and $t_{j}$. Let $K\left(s_{i}\right)=\left\{t_{j}\right.$ : $\left.\psi_{i}\left(s_{i}, t_{j}\right)>0\right\}$ be the interval of interest. Note first that when we increase $s_{i}$, the interval of interest can only expand, because $\psi_{i}\left(s_{i}, t_{j}\right)$ is non-decreasing in $s_{i}$.

Within interval $K\left(s_{i}\right)$, function $\psi_{i}\left(s_{i}, t_{j}\right)$ is positive and therefore equal to $\lambda_{i}\left(t_{j}\right) s_{i}+\gamma_{i}\left(t_{j}\right)$. Furthermore, weak monotonicity implies that as a function of $t_{j}, \psi_{i}\left(s_{i}, t_{j}\right)$ is a piecewise linear function with derivative (slope) in $\{0,1,-1\}$ (see for example, Figure 1).

The piecewise linear function $\psi_{i}\left(s_{i}, t_{j}\right)$ is differentiabl $f^{5}$ everywhere except perhaps of the at most two break points. We have

$$
\frac{\partial \psi_{i}\left(s_{i}, t_{j}\right)}{\partial t_{j}}=\frac{\partial \lambda_{i}\left(t_{j}\right)}{\partial t_{j}} s_{i}+\frac{\partial \gamma_{i}\left(t_{j}\right)}{\partial t_{j}} .
$$

If $\partial \lambda_{i}\left(t_{j}\right) / \partial t_{j} \neq 0$, then by varying $s_{i}$, the slope cannot stay in $\{0,1,-1\}$. We conclude that $\partial \lambda_{i}\left(t_{j}\right) / \partial t_{j}=0$ in each piece, which shows that $\lambda_{i}\left(t_{j}\right)$ is independent of $t_{j}$ within each piece and therefore independent of $t_{j}$ everywhere.

The following corollary is an essential tool for establishing the lower bound.

Corollary 1. The constant parts of the piecewise linear function $\psi_{i}\left(s_{i}, s_{-i}, t_{-i}\right)$, as a function of $t_{j}$, are independent of $s_{j}$, for $j \neq i$.

Proof. We fix all values except for tasks $i$ and $j$. By the previous lemma, when we change $s_{j}$, the boundary $\psi_{j}\left(s_{j}, s_{-j}, t_{-j}\right)$ is translated rectilinearly and therefore its break points remain the same. Since the constant parts of $\psi_{i}\left(s_{i}, s_{-i}, t_{-i}\right)$ are determined by the break points of $\psi_{j}\left(s_{j}, s_{-j}, t_{-j}\right)$, they also remain the same. See Figure 3 for an illustration.

Corollary 2. The piecewise linear function $\psi_{i}\left(s_{i}, s_{-i}, t_{-i}\right)$ is either non-decreasing in $t_{j}$ or non-decreasing in $s_{j}$.

Proof. When we fix all other values and consider the $t_{-\{i, j\}}$ cut, it is either quasi-flipping or crossing, in which case $\psi_{i}\left(s_{i}, s_{-i}, t_{-i}\right)$ is non-decreasing in $t_{j}$, or quasi-bundling, in which case $2 \mathrm{D}$ geometry shows that it is non-decreasing in $s_{j}$ (when $s_{j}$ decreases the diagonal part shifts downwards, and the rectilinear parts remain fixed). See Figure 3 for an illustration.

\subsection{The main theorem}

We now have most of the ingredients to prove next theorem which directly implies the main lower bound Theorem 1 ).

Theorem 3. The approximation ratio of linear truthful algorithms on restricted $(s, t)$ instances (Equation 2) with $n$ machines is at least $\sqrt{n-1}$.

To prove this theorem, we fix some linear truthful algorithm and we focus on a particular set of instances, which we call $s$-inefficient. The set of $s$-inefficient instances depends on the linear algorithm and each instance consists of two types of tasks: either a task is unimportant (i.e., it has very small value for one of the machines), or its $s$ value is significantly higher than its $t$ value, yet the algorithm allocates the task to the $s$-player.

\footnotetext{
${ }^{5}$ It is not hard to see that if $\psi_{i}\left(s_{i}, t_{j}\right)$ is differentiable then so are $\lambda_{i}\left(t_{j}\right)$ and $\gamma_{i}\left(t_{j}\right)$; alternatively, one could use small differences instead of derivatives.
} 


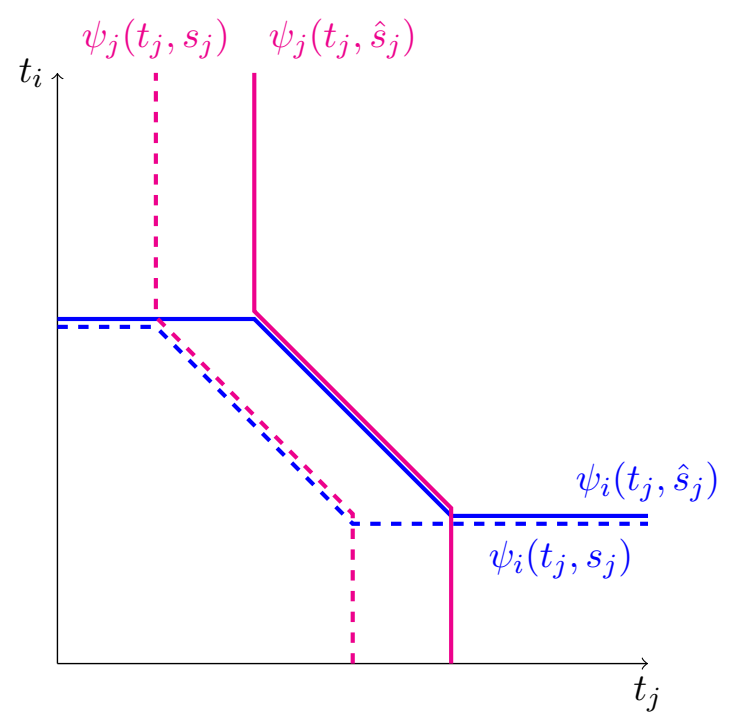

Figure 3: Consider the dashed lines that show $\psi_{i}\left(t_{j}, s_{j}\right)$ and $\psi_{j}\left(t_{j}, s_{j}\right)$ (these functions may depend on other values but they play no role and we can ingore them). The regular lines show $\psi_{i}\left(t_{j}, \hat{s}_{j}\right)$ and $\psi_{j}\left(t_{j}, \hat{s}_{j}\right)$, for some $\hat{s}_{j}>s_{j}$. Notice that linearity implies that $\psi_{i}\left(t_{j}, \hat{s}_{j}\right)$ is a shift to the right of $\psi_{i}\left(t_{j}, s_{j}\right)$; in particular, the break point stay at the same height. As a consequence, the blue horizontal parts remain at the same height and the blue diagonal part shifts to the right. Therefore $\psi_{i}\left(t_{j}, \hat{s}_{j}\right) \geq \psi_{i}\left(t_{j}, s_{j}\right)$.

Definition 9 (s-inefficient instances). Let's call a task $i$ trivial when either $s_{i}=0$ or $t_{i} \in$ $\left(0, \delta_{0}\right]$, for some fixed (sufficiently small) $\delta_{0}$. We call a restricted $(t, s)$ instance $s$-inefficient for a mechanism, if it contains at least one non-trivial task, every non-trivial task $i$ satisfies $s_{i} / t_{i}>\sqrt{n-1}$, and the mechanism allocates all non-trivial tasks to the $s$-player.

The heart of the proof is to show that if the set of s-inefficient instances is non-empty, there exists an s-inefficient instance with exactly one non-trivial task. From this, it immediately follows that the algorithm has approximation ratio at least $\sqrt{n-1}$.

However, it may be that for a given linear truthful algorithm there are no $s$-inefficient instances. But then we can use weak monotonicity to easily derive a $\sqrt{n-1}$ lower bound on the approximation ratio as the following lemma shows.

Lemma 5. If for a given truthful algorithm the set of s-inefficient instances is empty, then its approximation ratio is at least $\sqrt{n-1}$.

Proof. Towards a contradiction, consider an algorithm that has approximation ratio $\sqrt{n-1}-\delta$, for some $\delta>0$, for which the set of $s$-inefficient instances is empty. We consider the instance with $t_{i}=\alpha=1 / \sqrt{n-1}-\delta / n$ and $s_{i}=1$, for all $i \in[n-1]$.

$$
\left[\begin{array}{cccc}
\alpha & \alpha & \cdots & \alpha \\
1 & & & \\
& 1 & & \\
& & \vdots & \\
& & & 1
\end{array}\right]
$$

Note that at least one task is allocated to the $s$-players, because if all tasks are assigned to the $t$-player, the makespan is $(n-1) \alpha=\sqrt{n-1}-(n-1) \delta / n>\sqrt{n-1}-\delta$, the optimum makespan is 1 , and the approximation ratio is strictly greater than $\sqrt{n-1}-\delta$.

Now for every task $i$ which is allocated to the $t$-player, we lower its value from $\alpha$ to some small value in $\left(0, \delta_{0}\right]$ and increase the $t$ value of every other task to $\alpha+\delta /(2 n)<1 / \sqrt{n-1}$. By 


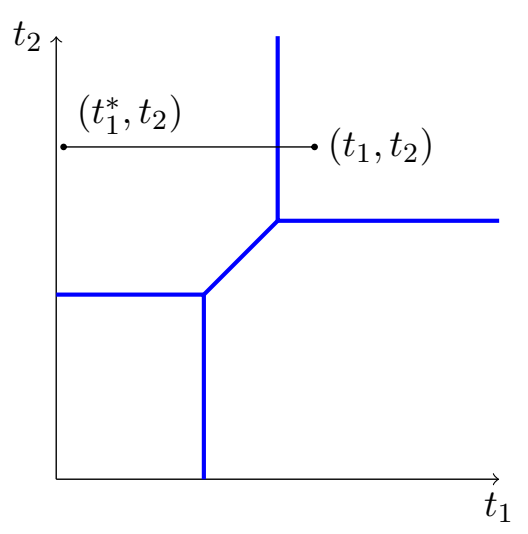

Figure 4: When the cut is quasiflipping and neither task is given to the $t$-player in the allocation for $\left(t_{1}, t_{2}\right)$, then the second task is still not allocated to the $t$-player in the allocation for $\left(t_{1} * \approx\right.$ $\left.0, t_{2}\right)$.

weak monotonicity (Lemma 2), the allocation of the tasks for the $t$-player remains the same. Since at least one task is allocated to the $s$-players, we end up with an $s$-inefficient instance, a contradiction.

The proof of the next lemma (Lemma 6), which provides a very useful property of linear mechanisms, is the most critical part of the proof. It shows that linear weakly monotone algorithms satisfy a locality property, that bears some resemblance to the locality property in [31]. But unlike [31, our proof does not assume this property, but it derives it from weak monotonicity for the special class of instances that we consider.

Lemma 6. If for a given linear truthful algorithm the set of s-inefficient instances is non-empty, then there is an s-inefficient instance with exactly one non-trivial task.

Proof. Fix a linear truthful algorithm and consider an $s$-inefficient instance $(t, s)$ with the minimum number of non-trivial tasks. If the number of non-trivial tasks is at least two, let us assume without loss of generality that tasks 1 and 2 are non-trivial. We will derive a contradiction by reducing the number of non-trivial tasks.

Consider the boundary function of the first task, $\psi_{1}\left(s_{1}, s_{-1}, t_{-1}\right)=\max \left(0, \lambda_{1}\left(s_{-1}, t_{-1}\right) s_{1}+\right.$ $\gamma_{1}\left(s_{-1}, t_{-1}\right)$, for some positive $\lambda_{1}$. The crux of the matter is that we can reduce the value of $s_{1}$ to 0 or $t_{1}$ to at most $\delta_{0}$, and guarantee that the $t$-player will keep not getting the second task. This guarantees that the second task is non-trivial and is given to the $s$-player, while the first task becomes trivial.

If $\psi_{2}\left(s_{2}, s_{-2}, t_{-2}\right)$ as a function of $t_{1}$ is non-decreasing (i.e., the $t_{-\{1,2\}}$ cut is quasi-flipping or crossing), we set $t_{1}^{*} \in\left(0, \delta_{0}\right]$. Since $\psi_{2}\left(s_{2}, s_{-2}, t_{-2}\right)$ is non-decreasing in $t_{1}$ (Corollary 2), in the new instance the second task is still allocated to the $s$-player (Figure 4).

Otherwise (i.e., the $t_{-\{1,2\}}$ cut is quasi-bundling), by Corollary 1 , function $\psi_{2}\left(s_{2}, s_{-2}, t_{-2}\right)$ is non-decreasing in $s_{1}$. In this case, we change the instance as follows (see Figures 5 for an illustration of the first two cases):

a) if $\gamma_{1}\left(s_{-1}, t_{-1}\right) \geq 0$, we set $s_{1}^{*}=0$ and $t_{1}^{*}=t_{1}$,

b) otherwise, if $\psi_{1}\left(s_{1}, s_{-1}, t_{-1}\right)>0$, we set $s_{1}^{*}=-\gamma_{1}\left(s_{-1}, t_{-1}\right) / \lambda_{1}\left(s_{-1}, t_{-1}\right)$ and $t_{1}^{*} \in\left(0, \delta_{0}\right]$, small enough to make task 1 trivial,

c) and if $\psi_{1}\left(s_{1}, s_{-1}, t_{-1}\right)=0$, we set $s_{1}^{*}=s_{1}$ and $t_{1}^{*} \in\left(0, \delta_{0}\right]$, small enough to make task 1 trivial. 

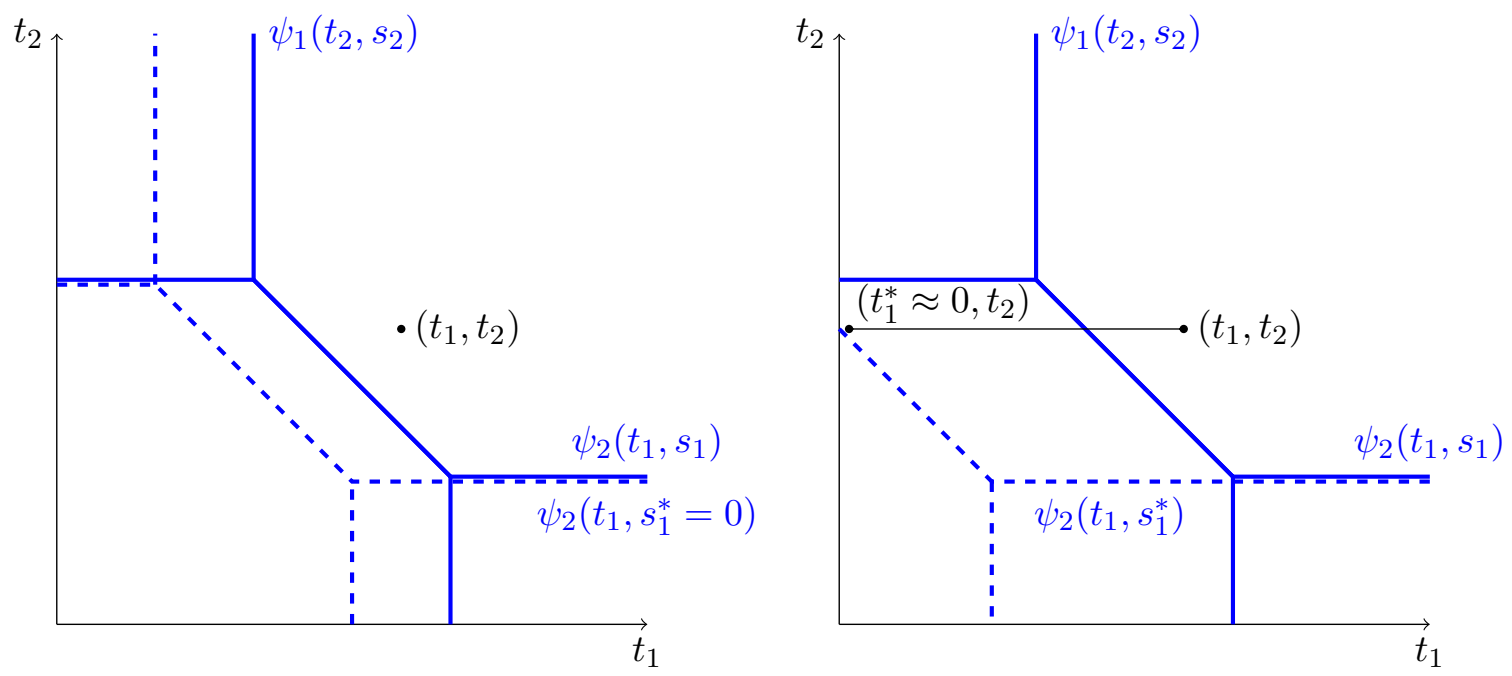

Figure 5: Left side case (a), when $s_{i} *$ decreases to 0 : the boundary $\psi_{2}\left(t_{1}, s_{1}^{*}\right)$ is lower than the boundary $\psi_{2}\left(t_{1}, s_{1}^{*}\right)$. Both tasks are still allocated to the $s$-player. Right side case (b), when the $s_{i}$ decreases to $s_{1}^{*}=-\gamma_{1}\left(s_{-1}, t_{-1}\right) / \lambda_{1}\left(s_{-1}, t_{-1}\right)$ and $t_{1}$ changes to $t_{1}^{*} \approx 0$. The new $\left(t_{1}^{*}, t_{2}\right)$ remains in the region in which both tasks are allocated to the $s$-player.

In the first two cases, we lower the $s_{1}$ value to $s_{1}^{*}$ until either $s_{1}^{*}$ becomes 0 (case a), or $\psi_{1}\left(s_{1}^{*}, s_{-1}, t_{-1}\right)$ becomes 0 and then we set $t_{1}=t_{1} * \approx 0$ (case b). The third case is when $\psi_{1}\left(s_{1}, s_{-1}, t_{-1}\right)$ is already 0 .

In all cases, the first task becomes a trivial task with the new values. Furthermore, in all cases the new value of $s_{1}$ is not greater than the original value: $s_{1}^{*} \leq s_{1}$. This is clearly true in the first and third case. To see that this is true in the second case, observe that the boundary $\psi_{1}\left(s_{1}, s_{-1}, t_{-1}\right)$, which is a non-decreasing function on $s_{1}$, moved from a positive value to $\psi_{1}\left(s_{1}^{*}, s_{-1}, t_{-1}\right)=0$.

We now argue that the second task is still given to the s-player after the change. The argument is based on Corollary 1, which guarantees that the changes can only shift the boundary $\psi_{2}\left(s_{2}, s_{-2}, t_{-2}\right)$ rectilinearly: in Figure 5 , the slanted part moves only horizontally.

For case (a), by Corollary 1 , the boundary $\psi_{2}\left(s_{2}, s_{-2}, t_{-2}\right)$ did not increase and therefore the second task is still given to the $s$-player (left part of Figure 5). For the other two cases, this is not sufficient because $t_{1}$ changed and therefore $t_{-2}$ changed as well. For case (b), the change shifts the slanted boundary (right part of Figure 5). In its new position, it crosses the boundary of the positive orthant at $\left(0, t_{2}\right)$ which is dominated by the point $\left(t_{1}^{*}, t_{2}\right)$. Therefore, the $t$-player gets neither task, and shows that the $s$-player gets the second task. Case (c) is simpler and similar to the second one; the difference is that the boundary starts at the leftmost position and it does not move at all (in the right part of Figure 5, the solid and dashed lines coincide and they may closer to the beginning of axes).

The change of values of the first task did not change the allocation of the second task, but it may have changed the allocation of the remaining non-trivial tasks. But we can use weak monotonicity to further change the instance to obtain an $s$-inefficient instance. If some nontrivial tasks changed allocation and were given to the $t$ player, we reduce their values to 0 . This will make them trivial tasks and, by weak monotonicity (Lemma 2), preserve the allocation of the first player for the other non-trivial task ${ }^{6}$. The resulting instance is $s$-inefficient and has fewer non-trivial tasks, a contradiction.

\footnotetext{
${ }^{6}$ Strictly speaking, to guarantee that the allocation remains the same for the non-trivial tasks, we need to increase slightly their $t$ value. This is possible without violating the constraint $s_{i} / t_{i}>\sqrt{n-1}$, which shows that the resulting instance is also $s$-inefficient.
} 
The proof of the main result of this section follows directly from the last two lemmas.

Proof of Theorem 3. By the last two lemmas, either a linear truthful algorithm has approximation ratio at least $\sqrt{n-1}$, or there exists an $s$-inefficient instance with one non-trivial task. The approximation ratio of such an instance is trivially at least $\sqrt{n-1}$, and the proof is complete.

\section{References}

[1] Aaron Archer and Robert Kleinberg. Truthful germs are contagious: A local to global characterization of truthfulness. In ACM Conference on Electronic Commerce (EC), 2008.

[2] Aaron Archer and Éva Tardos. Truthful mechanisms for one-parameter agents. In Proc. of the 42nd IEEE Symposium on Foundations of Computer Science (FOCS), pages 482-491, 2001.

[3] Itai Ashlagi, Shahar Dobzinski, and Ron Lavi. Optimal lower bounds for anonymous scheduling mechanisms. Mathematics of Operations Research, 37(2):244-258, 2012.

[4] Vincenzo Auletta, George Christodoulou, and Paolo Penna. Mechanisms for scheduling with single-bit private values. Theory Comput. Syst., 57(3):523-548, 2015.

[5] S. Bikhchandani, S. Chatterji, R. Lavi, A. Mu'alem, N. Nisan, and A. Sen. Weak monotonicity characterizes deterministic dominant strategy implementation. Econometrica, 74(4):1109-1132, 2006.

[6] Shuchi Chawla, Jason D. Hartline, David L. Malec, and Balasubramanian Sivan. Priorindependent mechanisms for scheduling. In Dan Boneh, Tim Roughgarden, and Joan Feigenbaum, editors, Symposium on Theory of Computing Conference, STOC'13, Palo Alto, CA, USA, June 1-4, 2013, pages 51-60. ACM, 2013.

[7] Xujin Chen, Donglei Du, and Luis Fernando Zuluaga. Copula-based randomized mechanisms for truthful scheduling on two unrelated machines. Theory Comput. Syst., 57(3):753$781,2015$.

[8] George Christodoulou, Elias Koutsoupias, and Annamária Kovács. Mechanism design for fractional scheduling on unrelated machines. ACM Transactions on Algorithms, 6(2), 2010.

[9] George Christodoulou, Elias Koutsoupias, and Angelina Vidali. A characterization of 2player mechanisms for scheduling. In Algorithms - ESA 2008, 16th Annual European Symposium, Karlsruhe, Germany, September 15-17, 2008. Proceedings, pages 297-307, 2008.

[10] George Christodoulou, Elias Koutsoupias, and Angelina Vidali. A lower bound for scheduling mechanisms. Algorithmica, 55(4):729-740, 2009.

[11] George Christodoulou and Annamária Kovács. A deterministic truthful ptas for scheduling related machines. SIAM J. Comput., 42(4):1572-1595, 2013.

[12] Edward H. Clarke. Multipart pricing of public goods. Public Choice, 8, 1971.

[13] Constantinos Daskalakis and S. Matthew Weinberg. Bayesian truthful mechanisms for job scheduling from bi-criterion approximation algorithms. In Piotr Indyk, editor, SODA, pages 1934-1952. SIAM, 2015.

[14] Peerapong Dhangwatnotai, Shahar Dobzinski, Shaddin Dughmi, and Tim Roughgarden. Truthful approximation schemes for single-parameter agents. SIAM J. on Computing, 40(3):915-933, 2011. 
[15] Shahar Dobzinski. Breaking the logarithmic barrier for truthful combinatorial auctions with submodular bidders. In Proceedings of the 48th Annual ACM SIGACT Symposium on Theory of Computing, STOC 2016, Cambridge, MA, USA, June 18-21, 2016, pages 940-948, 2016.

[16] Shahar Dobzinski and Noam Nisan. Multi-unit auctions: Beyond roberts. J. Economic Theory, 156:14-44, 2015.

[17] Shahar Dobzinski and Mukund Sundararajan. On characterizations of truthful mechanisms for combinatorial auctions and scheduling. In Proceedings 9th ACM Conference on Electronic Commerce (EC-2008), Chicago, IL, USA, June 8-12, 2008, pages 38-47, 2008.

[18] Shahar Dobzinski and Jan Vondrák. Impossibility results for truthful combinatorial auctions with submodular valuations. J. ACM, 63(1):5:1-5:19, 2016.

[19] Leah Epstein, Asaf Levin, and Rob van Stee. A unified approach to truthful scheduling on related machines. In Proc. of the Twenty-Fourth Annual ACM-SIAM Symposium on Discrete Algorithm (SODA), pages 1243-1252, 2013.

[20] Yiannis Giannakopoulos and Maria Kyropoulou. The VCG mechanism for bayesian scheduling. ACM Trans. Economics and Comput., 5(4):19:1-19:16, 2017.

[21] Theodore Groves. Incentives in teams. Econometrica, 41(4):617-631, 1973.

[22] Roberts Kevin. The characterization of implementable choice rules. Aggregation and Revelation of Preferences, pages 321-348, 1979.

[23] Elias Koutsoupias and Angelina Vidali. A lower bound of $1+\phi$ for truthful scheduling mechanisms. Algorithmica, pages 1-13, 2012.

[24] Ron Lavi and Chaitanya Swamy. Truthful mechanism design for multidimensional scheduling via cycle monotonicity. Games and Economic Behavior, 67(1):99-124, 2009.

[25] Benny Lehmann, Daniel J. Lehmann, and Noam Nisan. Combinatorial auctions with decreasing marginal utilities. Games and Economic Behavior, 55(2):270-296, 2006.

[26] Stefano Leucci, Akaki Mamageishvili, and Paolo Penna. No truthful mechanism can be better than $n$ approximate for two natural problems. Games and Economic Behavior, 111:64-74, 2018.

[27] Pinyan Lu. Internet and network economics, 5th international workshop, wine 2009, rome, italy, december 14-18, 2009. proceedings. In Proc. of the 5th International Workshop on Internet and Network Economics (WINE), volume 5929 of Lecture Notes in Computer Science, pages 30-41. Springer, 2009.

[28] Pinyan Lu and Changyuan Yu. An improved randomized truthful mechanism for scheduling unrelated machines. In 25th Annual Symposium on Theoretical Aspects of Computer Science (STACS), volume 1 of LIPIcs, pages 527-538, 2008.

[29] Pinyan Lu and Changyuan Yu. Randomized truthful mechanisms for scheduling unrelated machines. In 4th International Workshop on Internet and Network Economics (WINE), pages 402-413, 2008.

[30] Ahuva Mu'alem and Michael Schapira. Setting lower bounds on truthfulness. Games and Economic Behavior, 110:174-193, 2018.

[31] Noam Nisan and Amir Ronen. Algorithmic mechanism design. Games and Economic Behavior, 35:166-196, 2001. 
[32] Michael E. Saks and Lan Yu. Weak monotonicity suffices for truthfulness on convex domains. In Proceedings 6th ACM Conference on Electronic Commerce (EC), pages 286-293, 2005 .

[33] William Vickrey. Counterspeculations, auctions and competitive sealed tenders. Journal of Finance, 16:8-37, 1961.

[34] Changyuan Yu. Truthful mechanisms for two-range-values variant of unrelated scheduling. Theoretical Computer Science, 410(21-23):2196-2206, 2009. 


\section{Appendix: All truthful mechanisms for 2 tasks and 2 machines}

\section{A Additive valuations for 2 tasks and 2 machines}

\section{A.1 Basic concepts and notation}

In this section we characterize WMON allocations for two tasks and two players (called $t$-player, and $s$-player) with additive valuations $t=\left(t_{1}, t_{2}\right)$, and $s=\left(s_{1}, s_{2}\right)$, so that both $s_{1}$ and $s_{2}$ are bounded by an arbitrarily large but fixed value $B$.

Let $(A, P)$ be a truthful mechanism, where $A$ is the WMON allocation function, and $P$ denotes the payment function. For input $(t, s)$ the allocation is $A(t, s)$. Since we have only tasks 1 and 2 , in $A(t, s)$ we can denote the allocation to one of the players as $\alpha_{t}, \alpha_{s} \in\{12,1,2, \emptyset\}$. We often abuse notation and identify $A(t, s)$ by the allocation $\alpha_{t}$ of the $t$-player; e.g., we say that the allocation $A(t, s)$ is 12 , meaning that $\alpha_{t}=12$, and $\alpha_{s}=\emptyset$.

For given $s \in[0, B) \times[0, B)$ the allocation for the $t$-player as function of his bids $\left(t_{1}, t_{2}\right)$ is denoted by $A[s]$, and symmetrically $A[t]$ is an allocation function for the $s$-player. For $\alpha_{t} \in\{12,1,2, \emptyset\}$, the allocation regions $R_{\alpha_{t}}(s) \subseteq \mathbb{R}_{>0}^{2}$ of $A[s]$ are defined to be the interior (wrt. $\mathbb{R}_{\geq 0}^{2}$ ) of the set of all $t$ values such that $A(t, s)=\alpha_{t}$. (For the $s$-player we denote the respective regions by $R_{\alpha_{s}}^{s}(t)$. ) We assume that for every $s \in[0, B) \times[0, B)$ there exist $t$ values so that the $t$-player receives no task, i.e., that $R_{\emptyset}(s) \neq \emptyset$ for every $s$. Note that we do not assume the same for the $s$-player, because his bids are bounded. We remark that the assumption $R_{\emptyset}(s) \neq \emptyset$ does not restrict the types of possible WMON mechanisms, but it simplifies the characterization to a large extent.

It is known that in the case of two tasks, the regions in a WMON allocation subdivide $R_{\geq 0}^{2}$ basically in three possible forms, which will turn out to be characteristic for the type of the whole allocation-function $A$. (see Figure 6) The regions and their boundaries determine the critical values for $t_{1}$ (in fact, as function of $t_{2}$ ) above which the $t$-player cannot get task 1 , and symmetrically for task 2 . These critical value functions are ultimately determined by the payment functions $P_{\emptyset}(s)=0, P_{1}(s), P_{2}(s), P_{12}(s)$ for the fixed $s$.

For any given $s$, we assume w.l.o.g. virtual payments for the $t$-player, which are the unique normalized and monotone (as set functions) payments for a given allocation $A[s]$ (see also Section B):

Definition 10. Let $(A, \hat{P})$ be a truthful mechanism, and $s=\left(s_{1}, s_{2}\right)$ be fixed. The payments occuring in this definition are all defined for this given $s$, so we omit $(s)$ from the notation. Since $R_{\emptyset} \neq \emptyset$, we can assume w.l.o.g. that the payment function $\hat{P}$ is normalized, that is, $\hat{P}_{\emptyset}=0$. We define the virtual payments as:

$$
\begin{gathered}
P_{\emptyset}=0, \\
P_{1}=\max \left\{\hat{P}_{1}, 0\right\}, \quad P_{2}=\max \left\{\hat{P}_{2}, 0\right\}, \\
P_{12}=\max \left\{\hat{P}_{12}, P_{1}, P_{2}\right\} .
\end{gathered}
$$

The virtual payments are always well-defined, non-negative, and truthful payments to the $t$-player for the given allocation $A[s]$.

For fixed $s$, the virtual payments determine uniquely the allocation and vice-versa. The value $P_{1}$ determines the position of the vertical boundary/critical value between $R_{\emptyset}$ and $R_{1}$. Since $R_{\emptyset}$ is nonempty, this boundary always exists; if $R_{1}=\emptyset$, then $P_{1}=0$. Analogously, $P_{2}$ is the position of the horizontal boundary between $R_{\emptyset}$ and $R_{2}$; furthermore $P_{12}-P_{2}$ (resp. $P_{12}-P_{1}$ ) is the position of the vertical (horizontal) boundary between $R_{12}$ and $R_{2}$ (resp. $R_{12}$ 


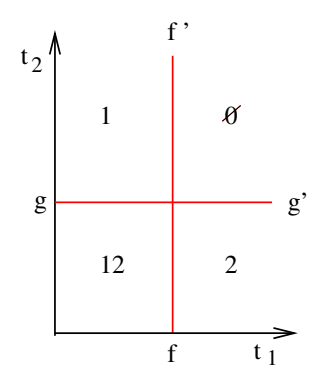

(a)

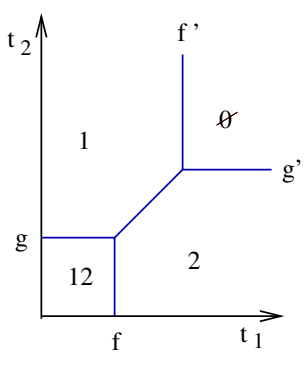

(b)

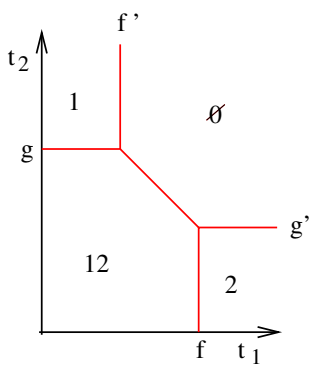

(c)

Figure 6: The allocations to a single player depending on his own 2-dimensional bid vector partition the bid-space according to one of these three shapes (where the interior of some region(s) different from $R_{\emptyset}$ may be empty). The allocation $A[s]$ is called (a) crossing, (b) quasi-flipping, or (c) quasi-bundling, respectively.

and $R_{1}$ ) if these regions are nonempty. In the proof below, for given $s$ we also use the short notation

$$
f^{\prime}=P_{1}, \quad g^{\prime}=P_{2},
$$

and if the respective boundary exists then also

$$
f=P_{12}-P_{2}, \quad g=P_{12}-P_{1} .
$$

Definition 11. For given $s$, we call the allocation $A[s]$

- quasi-bundling, if there are at least two points $t \neq t^{\prime}$ on the boundary of $R_{12}$ and $R_{\emptyset}(\Leftrightarrow$ $\left.P_{1}<P_{12}-P_{2}\right)$

- quasi-flipping, if there are at least two points $t \neq t^{\prime}$ on the boundary of $R_{1}$ and $R_{2}$ $\left(\Leftrightarrow P_{1}>P_{12}-P_{2}\right)$.

- crossing otherwise ( $\Leftrightarrow P_{1}=P_{12}-P_{2}$ ) (see also Figure 6)

Next we introduce the four different types of WMON allocations that can occur in the above setting. After that we prove the characterization $\sqrt[7]{7}$

\section{A.1.1 Relaxed task-independent allocations}

An allocation function $A$ is task-independent if, for $i \in\{1,2\}$, the allocation of task $i$ depends only on the input values $s_{i}$ and $t_{i}$. For the $t$-player, the critical-value of $t_{1}$, i.e., the lowest value, above which $t_{1}$ does not get task 1 , is determined by an arbitrary, increasing function $\phi:[0, B) \rightarrow[0, \infty)$ of $s_{1}$. Analogously we can define the critical-value function $\eta\left(s_{2}\right)$ as the lowest value, depending on $s_{2}$, above which $t_{2}$ does not get task 28 Geometrically, in a taskindependent mechanism, the allocations $A[s]$ and $A[t]$ of both players are always crossing. In a relaxed task-independent mechanism the latter property is fulfilled in all but countably many $s$ (resp. $t$ ) points, in which both $\phi$ and $\eta$ (resp. $\phi^{-1}$ and $\eta^{-1}$ ) have a jump discontinuity:

Definition 12. An allocation is a relaxed task-independent allocation if there exist arbitrary, increasing critical-value functions for the $t$-player: $\phi\left(s_{1}\right)$ for task 1 , and $\eta\left(s_{2}\right)$ for task 2 , so that for every $s \in[0, B) \times[0, B)$

\footnotetext{
${ }^{7}$ Observe that there exist mechanisms that adhere to more than one of the given types.

${ }^{8}$ The tie-breaking (i.e., when e.g., $\left.t_{1}=\phi\left(s_{1}\right)\right)$ may in all cases depend on the other variables $\left(s_{2}\right.$ and $\left.t_{2}\right)$.
} 
- if $\phi()$ is continuous in the point $s_{1}$ OR $\eta()$ is continuous in the point $s_{2}$ then the allocation of the two tasks is independent and adheres to the critical values $\phi\left(s_{1}\right)$ and $\eta\left(s_{2}\right)$, respectively; (note however that if, e.g., $\eta$ has a jump discontinuity in $s_{2}$, then the critical value of for task 2 can depend on $s_{1}$, as long as it is between $\eta\left(s_{2}^{-}\right)$and $\eta\left(s_{2}^{+}\right)$);

- if $\phi()$ has a jump discontinuity in the point $s_{1}$ AND $\eta()$ has a jump discontinuity in $s_{2}$, then the allocation $A[s]$ of the $t$-player can be an arbitrary crossing, quasi-flipping, or quasi-bundling allocation, such that all critical values for task 1 are at least $\phi\left(s_{1}^{-}\right)$and at most $\phi\left(s_{1}^{+}\right)$, and similarly for task 2 .

Symmetric statements hold for the $s$-player with the critical-value-functions $\phi^{-1}\left(t_{1}\right)$ and $\eta^{-1}\left(t_{2}\right)$. In summary, every relaxed task-independent allocation is identical with a task-independent allocation on $t_{i} \in[0, \infty) \backslash T_{i}, s_{i} \in[0, B) \backslash S_{i}$, where the $T_{1}, T_{2}, S_{1}, S_{2}$ are countable sets.

\section{A.1.2 1-dimensional mechanisms}

In a one-dimensional mechanism at most two possible allocations are ever realized. The criticalvalue function between the two possible allocations is an arbitrary increasing function of the respective input variables.

Because of the assumption that $R_{\emptyset}(s) \neq \emptyset$ for every $s$, one of the occuring allocations of the $t$-player must be the allocation $\emptyset$. If the two occuring allocations (for the $t$-player) are $\emptyset$ and 12 , we call the mechanism bundling mechanism. In the other cases when 1 , and $\emptyset$; or when 2 , and $\emptyset$ are the possible allocations of the $t$-player, the mechanism is identical with a degenerate task-independent mechanism, where $\phi \equiv 0$ or $\eta \equiv 0$. Therefore we define only the case of a bundling mechanism in detail. We note that any bundling mechanism can be considered as a relaxed affine minimizer, with $\gamma_{1}=\infty$ and $\gamma_{2}=\infty$, but it will be convenient to treat bundling mechanisms separately.

Definition 13. In a bundling mechanism only the allocations $\emptyset$ and 12 can occur. There is an arbitrary, increasing function $\xi:[0, B) \rightarrow[0, \infty)$ so that $\xi\left(s_{1}+s_{2}\right)$ is the lowest value such that if $t_{1}+t_{2}>\xi\left(s_{1}+s_{2}\right)$ then the $t$-player gets $\emptyset$.

If $\xi$ has a jump discontinuity in some point $s_{1}+s_{2}$ then the critical value may depend on the concrete $\left(s_{1}, s_{2}\right)$ with the given fixed sum, as long as it is between $\xi\left(\left(s_{1}+s_{2}\right)^{-}\right)$and $\xi\left(\left(s_{1}+s_{2}\right)^{+}\right)$.

\section{A.1.3 Relaxed affine minimizers}

Definition 14. An allocation $A$ is an affine minimizer, if there exist positive constants per player $\mu_{t}$ and $\mu_{s}$, and constants $\gamma_{\emptyset}, \gamma_{12}, \gamma_{1}, \gamma_{2} \in \mathbb{R} \cup\{-\infty, \infty\}$ per allocation (say, of the $t$ player), so that for every input $(t, s)$ the allocation $A(t, s)$ minimizes over

$$
\mu_{t}\left(t_{1}+t_{2}\right)+\gamma_{12}, \quad \mu_{t} \cdot t_{1}+\mu_{s} \cdot s_{2}+\gamma_{1}, \quad \mu_{s} \cdot s_{1}+\mu_{t} \cdot t_{2}+\gamma_{2}, \quad \mu_{s}\left(s_{1}+s_{2}\right)+\gamma_{\emptyset} .
$$

In some affine minimizers, if $s_{1}+s_{2}$ and $t_{1}+t_{2}$ are both 'small', then the mechanism locally 'looks like' a bundling mechanism, with only the regions $R_{\emptyset}$ and $R_{12}$ for both players. If this is the case for all $\left(s_{1}, s_{2}\right)$ and all $\left(t_{1}, t_{2}\right)$ with sums $s_{1}+s_{2}<D$ and $t_{1}+t_{2}<C$ for some given $C, D>0$, then the mechanism becomes locally a bundling mechanism. This is formulated more precisely in the next definition:

Definition 15. An allocation $A$ is a relaxed affine minimizer, if there exist positive constants per player $\mu_{t}$ and $\mu_{s}$, and constants $\gamma_{\alpha}$ per allocation $\alpha$ (of the $t$-player), furthermore an arbitrary increasing function $\xi:\left[0, \min \left(\gamma_{1}, \gamma_{2}\right)-\gamma_{\emptyset}\right) \rightarrow[0, \infty)$ (if the interval $\left[0, \min \left(\gamma_{1}, \gamma_{2}\right)-\gamma_{\emptyset}\right)$ is nonempty) with $\min \left(\gamma_{1}, \gamma_{2}\right)-\gamma_{12}=\xi\left(\min \left(\gamma_{1}, \gamma_{2}\right)-\gamma_{\emptyset}\right)$, so that for every input $(t, s)$

a) if $\mu_{s} \cdot\left(s_{1}+s_{2}\right) \geq \min \left(\gamma_{1}, \gamma_{2}\right)-\gamma_{\emptyset}$, the allocation $A(t, s)$ is that of an affine minimizer with the given constants 
b) if $\mu_{s} \cdot\left(s_{1}+s_{2}\right) \leq \min \left(\gamma_{1}, \gamma_{2}\right)-\gamma_{\emptyset}$, then if $\mu_{t} \cdot\left(t_{1}+t_{2}\right)>\xi\left(\mu_{s} \cdot\left(s_{1}+s_{2}\right)\right)$ then the allocation for the $t$-player is $\emptyset$ and if $\mu_{t} \cdot\left(t_{1}+t_{2}\right) \leq \xi\left(\mu_{s} \cdot\left(s_{1}+s_{2}\right)\right)$ then it is 12 .

The condition $\min \left(\gamma_{1}, \gamma_{2}\right)-\gamma_{12}=\xi\left(\min \left(\gamma_{1}, \gamma_{2}\right)-\gamma_{\emptyset}\right)$ is required so that the affine minimizer (a) is appropriately 'glued' to the bundling mechanism (b).

\section{A.1.4 Constant mechanisms}

In a constant mechanism the allocation is independent of the bids of at least one of the players. This property can also be interpreted as being an affine minimizer with multiplicative constant $\mu=0$. Since we are interested in non-constant critical value functions, it will be convenient to treat constant mechanisms separately from affine minimizers.

\section{A.2 The main result}

Theorem 4. Every WMON allocation for two tasks and two additive players with bids $t \in$ $(0, \infty) \times(0, \infty)$ and $s \in(0, B) \times(0, B)$, where both tasks are always allocated, and $R_{\emptyset}(s) \neq \emptyset$ for every $s$, is one of these four types: (1) relaxed affine minimizer, (2) relaxed task-independent mechanism (3) one-dimensional mechanism, or (4) constant mechanism.

\section{A.3 Proof of Theorem 4}

Notation. For the critical values (boundary positions) of the $t$-player for given $s \in[0, B) \times[0, B)$ we use the notation $f^{\prime}(s)=P_{1}(s), g^{\prime}(s)=P_{2}(s)(s$ is omitted from the notation if it is clear from the context).

The critical values $f^{\prime}, g^{\prime} \in[0, \infty)$ are always defined and finite, since $f^{\prime}=\infty$ or $g^{\prime}=\infty$ would mean $R_{\emptyset}=\emptyset$.

We prove next, that $f^{\prime}$ is an increasing function of $s_{1}$, furthermore it is continuous in almost every (i.e., up to at most countably many) point $s_{1} \in[0, B)$, and in every $s_{1}$ where $f^{\prime}$ is continuous, it is independent of $s_{2}$. Symmetric statements hold for $g^{\prime}$.

Lemma 7. $f^{\prime}$ is monotone increasing in $s_{1}$ in the following strong sense: Let $s_{2}, s_{2}^{\prime}$ be arbitrary. If $s_{1}<s_{1}^{\prime}$ then $f^{\prime}\left(s_{1}, s_{2}\right) \leq f^{\prime}\left(s_{1}^{\prime}, s_{2}^{\prime}\right)$.

Proof. Let $s=\left(s_{1}, s_{2}\right)$, and $s^{\prime}=\left(s_{1}^{\prime}, s_{2}^{\prime}\right)$, and assume for contradiction that $f^{\prime}(s)>f^{\prime}\left(s^{\prime}\right)$. Then there exists a $\hat{t}$ (with high enough $\hat{t}_{2}$ ) so that $\hat{t} \in R_{1}(s)$ but $\hat{t} \in R_{\emptyset}\left(s^{\prime}\right)$. In turn, in the allocation $A[\hat{t}]$ the $s$-player gets both tasks if he bids $s^{\prime}$, and he gets only task 2 with bid $s$, contradicting WMON for the $s$-player, because $s_{1}<s_{1}^{\prime}$ (cf. Figure 6).

Since $f^{\prime}\left(., s_{2}\right)$ is increasing as a function of $s_{1}$, it must be continuous in all but at most countably many points $s_{1}$. Furthermore, since $f^{\prime}$ is increasing independently of $s_{2}$, it must be independent of $s_{2}$ in all $s_{1}$ where it is continuous, as we show next.

Lemma 8. If $f^{\prime}$ is continuous as a function of $s_{1}$ in some point $\left(\bar{s}_{1}, \bar{s}_{2}\right)$, then it is independent of $s_{2}$, i.e., then $f^{\prime}\left(\bar{s}_{1}, s_{2}\right)=f^{\prime}\left(\bar{s}_{1}, \bar{s}_{2}\right)$ for every $s_{2}$.

Proof. Let $f^{\prime}\left(., \bar{s}_{2}\right)$ be continuous as a function of $s_{1}$ in the point $\bar{s}_{1}$. Assume for contradiction w.l.o.g., that $f^{\prime}\left(\bar{s}_{1}, \bar{s}_{2}\right)>f^{\prime}\left(\bar{s}_{1}, s_{2}\right)$ for some $s_{2} \neq \bar{s}_{2}$. Since $f^{\prime}\left(., \bar{s}_{2}\right)$ is continuous in the point $\bar{s}_{1}$, we can take a $\bar{s}_{1}-\delta$, so that $f^{\prime}\left(\bar{s}_{1}-\delta, \bar{s}_{2}\right)>f^{\prime}\left(\bar{s}_{1}, s_{2}\right)$ also holds. However, this contradicts Lemma 7 , because $\bar{s}_{1}-\delta<\bar{s}_{1}$.

We introduce similar notation for other critical values of the $t$-player based on the virtual payments.

Notation. If $R_{12}(s) \neq \emptyset$ and $R_{2} \neq \emptyset$, OR $P_{12}-P_{2}=0$, then let $f(s)=P_{12}-P_{2}$. Symmetrically, if $R_{12}(s) \neq \emptyset$ and $R_{1} \neq \emptyset$, OR $P_{12}-P_{1}=0$, then let $g(s)=P_{12}-P_{1}$. 
As opposed to $f^{\prime}$ and $g^{\prime}$, the values $f$ and $g$ are not defined for every $s \in[0, B) \times[0, B)$; this is needed for the following lemmas to hold. Note however that the only case when neither $f$, nor $g$ are defined is, when $f^{\prime}=g^{\prime}=0$, and the allocation is quasi-bundling. The proofs of Lemmas 9 and 10 are somewhat more involved variants of those of Lemmas 7 and 8 , and are deferred to the full version.

Lemma 9. $f$ is monotone increasing in $s_{1}$ in the following sense: Let $s_{1} \leq s_{1}^{\prime}$ and $s_{2}<s_{2}^{\prime}$. If $f$ is defined for $s=\left(s_{1}, s_{2}\right)$, then $f$ is also defined for $s^{\prime}=\left(s_{1}^{\prime}, s_{2}^{\prime}\right)$. Furthermore $f\left(s_{1}, s_{2}\right) \leq f\left(s_{1}^{\prime}, s_{2}^{\prime \prime}\right)$, whenever $s_{1}<s_{1}^{\prime}$, and $f$ is defined in $\left(s_{1}^{\prime}, s_{2}^{\prime \prime}\right)$. Symmetric statements hold for $g$.

Lemma 10. If $f$ is continuous as a function of $s_{1}$ in some point $\left(\bar{s}_{1}, \bar{s}_{2}\right)$, then it is independent of $s_{2}$, i.e., then $f^{\prime}\left(\bar{s}_{1}, s_{2}\right)=f^{\prime}\left(\bar{s}_{1}, \bar{s}_{2}\right)$ for every $s_{2}$ where $f$ is defined, in particular for every $s_{2}>\bar{s}_{2}$.

We will sometimes abuse notation and consider $f$ and $f^{\prime}$ as univariate functions of $s_{1}$. This is incorrect only for at most countably many $s_{1}$ where these functions have jump discontinuities. In these $s_{1}$ the above single-variate functions remain undefined, or can be identified with, e.g., $\lim _{x \rightarrow s_{1}^{-}} f(x)$. Analogously we will often treat $g^{\prime}$ and $g$ as univariate functions.

Intuition. CASE A. below treats all the cases when for some $s$ an allocation function of the $t$-player exists, where at least three (interiors of) regions $R$ are nonempty, the allocation is quasi-flipping or quasi-bundling, and one of the critical value functions $f^{\prime}, g^{\prime}, f, g$ is continuous in $s$ as univariate function. In this case the WMON allocation function over the whole additive domain with bounded $s$, is a relaxed affine minimizer. The proof of this is based on three observations, roughly summarized as follows:

First, that once an allocation of the $t$-player is quasi-bundling (quasi-flipping) in some $s$, where at least one of $f, g, f^{\prime}, g^{\prime}$ is continuous as univariate function, we can infinitesimally adjust $s$ in a carefully selected direction, so that the allocation remains quasi-bundling (quasi-flipping), and all of these functions are continuous (when defined) in the adjusted $s$.

Second, that if in any quasi-flipping or quasi-bundling allocation all the critical value functions $f^{\prime}, g^{\prime}, f, g$ are continuous in some point $s$, then they all must have the same derivative in this point. This will follow from the WMON property of the $s$-player.

Third, that in such points $f^{\prime}$ and $f$ (if defined) are independent of $s_{2}$, and $g^{\prime}$, and $g$ are independent of $s_{1}$, which eventually implies that the above derivatives must remain the same for every s, implying that each of these critical value functions is linear.

In CASE B. a quasi-flipping allocation exists, but $f^{\prime} \equiv g^{\prime} \equiv 0$ (for every $s$ ), and therefore the regions $R_{1}(s)=R_{2}(s)=\emptyset$ for every $s$. In this case the allocation is a 1-dimensional monotone allocation over the sets of positive $s$ and $t$, where the two tasks are bundled and always allocated to the same player.

Finally, in CASE C. we consider those allocations where the $t$-player has a crossing figure whenever any of $f^{\prime}, g^{\prime}$ (or $f, g$ ) is continuous in $s$. In this case the mechanism is a relaxed task-independent mechanism.

We remark that if in CASE A. the common derivatives of $f, g, f^{\prime}, g^{\prime}$ happen to be 0 , or in CASES B. or C. the arbitrary increasing critical value functions turn out to be constant, then we obtain a constant mechanism. We do not discuss these cases separately in the rest of the proof.

We start the argument with three lemmas, stating that the mechanism must be a (relaxed) affine minimizer, if a single point $\hat{s}$ exists where all of $f^{\prime}, g^{\prime}$ and $f, g$ (if defined) are continuous as univariate functions (i.e., $f^{\prime}$ and $f$ in the point $\hat{s}_{1}$ and $g^{\prime}$ and $g$ in the point $\hat{s}_{2}$ ), and the allocation $A[\hat{s}]$ is quasi-flipping or quasi-bundling.

Lemma 11. Assume that both $f$ and $g$ are undefined or zero in every $s \in(0, B) \times(0, B)$. If an $\hat{s} \in(0, B) \times(0, B)$ exists so that the allocation $A[\hat{s}]$ of the t-player is quasi-flipping, $f^{\prime}>0$ is 


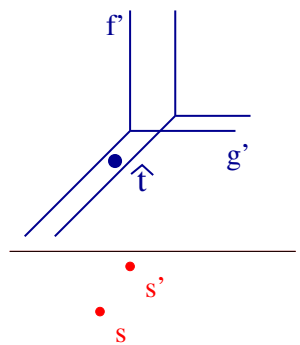

(a)

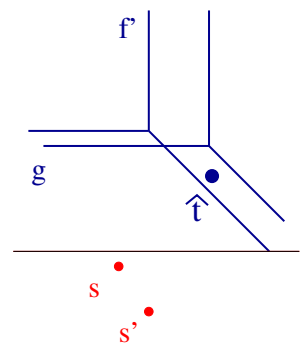

(b)

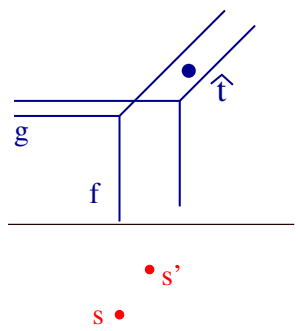

(c)

Figure 7: Illustrations to the proofs of Claims 1, 2, and 3

continuous in the point $\hat{s}_{1}$, and $g^{\prime}>0$ is continuous in the point $\hat{s}_{2}$, then the mechanism is an affine minimizer (with $\gamma_{12}=\infty$ ) for nonzero $t$ and $s$.

Proof. The proof makes use of the following claim, which is the core observation implying the linearity of the payment functions whenever an allocation is non-crossing:

Claim 1. Assume that $f^{\prime}>0$ is continuous as univariate function in the points $s_{1}$ and $s_{1}+\delta$, and $g^{\prime}>0$ is continuous in the points $s_{2}$, and $s_{2}+\delta$, furthermore the allocation of the $t$-player is quasi-flipping in a $2 \delta$-neighborhood of $s=\left(s_{1}, s_{2}\right)$. Then $f^{\prime}\left(s_{1}+\delta\right)-f^{\prime}\left(s_{1}\right)=g^{\prime}\left(s_{2}+\delta\right)-g^{\prime}\left(s_{2}\right)$.

Proof. (Figure 7 (a)) Assume for contradiction w.l.o.g. that $f^{\prime}\left(s_{1}+\delta\right)-f^{\prime}\left(s_{1}\right)>g^{\prime}\left(s_{2}+\delta\right)-$ $g^{\prime}\left(s_{2}\right)$. Since $f^{\prime}$ is continuous in $s_{1}+\delta$, it even holds that $f^{\prime}\left(s_{1}+\delta^{\prime}\right)-f^{\prime}\left(s_{1}\right)>g^{\prime}\left(s_{2}+\delta\right)-g^{\prime}\left(s_{2}\right)$ for some $\delta^{\prime}<\delta$.

Let $s^{\prime}=\left(s_{1}+\delta^{\prime}, s_{2}+\delta\right)$. Now there exists a point $\hat{t}$ so that $\hat{t} \in R_{2}(s)$ but $\hat{t} \in R_{1}\left(s^{\prime}\right)$. In turn, for this bid $\hat{t}$ of the $t$-player the $s$-player receives task 1 if he bids $s$, and he receives task 2 if he bids $s^{\prime}$. This contradicts WMON for the $s$-player because $s_{1}^{\prime}-s_{1}=\delta^{\prime}<\delta=s_{2}^{\prime}-s_{2}$.

By assumption of the lemma, $f$ or $g$ are never (defined and) positive for any $s \in(0, B) \times$ $(0, B)$. Therefore, the allocation is quasi-flipping for every $s \in(0, B) \times(0, B)$ for which $f^{\prime}(s)>0$ and $g^{\prime}(s)>0$.

Let us now fix $\bar{s}_{1}$, and $\delta=1$. Since $g^{\prime}$ has a jump in at most countably many points, we can pick a $\bar{s}_{2}$ so that $g^{\prime}$ is continuous in $\bar{s}_{2}+q$ whenever $q \in \mathbb{Q}$ and $\left(\bar{s}_{2}+q\right) \in(0, B)$. Restrict now $g^{\prime}$ on all such $s_{2}=\bar{s}_{2}+q$ points. For the constant $\Delta=f^{\prime}\left(\bar{s}_{1}+1\right)-f^{\prime}\left(\bar{s}_{1}\right)$ Claim 1 implies for the restricted $g^{\prime}$ that

$$
g^{\prime}\left(s_{2}+1\right)=g^{\prime}\left(s_{2}\right)+\Delta
$$

for every $s_{2}$ in the restricted set. Further, by taking $\delta=1 / 2$, we also obtain $g^{\prime}\left(s_{2}+1 / 2\right)=$ $g^{\prime}\left(s_{2}\right)+\Delta / 2$, and analogously

$$
g^{\prime}\left(s_{2}+1 / 2^{r}\right)=g^{\prime}\left(s_{2}\right)+\Delta / 2^{r}
$$

over all $s_{2}$ in the restriction. By taking the limit $r \rightarrow \infty$, we obtain that the restriction of $g^{\prime}$ is linear. Then, using that $g^{\prime}$ is increasing, and the set of $\bar{s}_{2}+q$ points is dense in $(0, B)$ we obtain that $g^{\prime}$ must be truncated linear over all $s_{2}>0$. As a further consequence $g^{\prime}$ is continuous, and independent of $s_{1}$. (The short proof presented here does not exclude that $g^{\prime}$ jumps to 0 in a certain $s_{2}$. An argument as in the proof of Claim 1 can exclude this.)

By analogous argument we obtain that $f^{\prime}$ is independent of $s_{2}$ and linear over all $s_{1}>0$ for which $f^{\prime}\left(s_{1}\right)>0$. Moreover, by Claim $1 f^{\prime}$ has the same multiplicative constant $\mu$ as $g^{\prime}$.

In summary, for $s>0$, for the virtual payments of the $t$-player we obtain $P_{1}(s)=f^{\prime}(s)=$ $\max \left(\mu \cdot s_{1}+\gamma_{1}, 0\right)$, and $P_{2}(s)=g^{\prime}(s)=\max \left(\mu \cdot s_{2}+\gamma_{2}, 0\right)$, and $P_{12}(s)=\max \left(P_{1}(s), P_{2}(s)\right)$ follows from $f=0$ or $g=0$. 
(We still need to exclude that the allocation is fully bundling for some $s$; however this is easy to prove, because the region $R_{12}$ could only increase with increasing $s$ and could not disappear in $\bar{s}$ and in every higher $s$.) The described virtual payments define an affine minimizer over $t \in(0, \infty) \times(0, \infty)$ and $s \in(0, B) \times(0, B)$. If $\mu=0$, then the mechanism is a constant mechanism (the allocation is independent of $s$ ). If $t_{i}=0$ or $s_{i}=0$, then the allocation can be different from that of an affine minimizer.

Lemma 12. Assume that $g^{\prime}\left(s_{2}\right)=0$ for every $s_{2} \in[0, B)$. If an $\hat{s} \in(0, B) \times(0, B)$ exists so that the allocation $A[\hat{s}]$ of the t-player is quasi-bundling, $f^{\prime}>0$ is continuous in the point $\hat{s}_{1}$, and $g>0$ is continuous in the point $\hat{s}_{2}$, then the mechanism is a relaxed affine minimizer (with $\left.\gamma_{2}=\infty\right)$.

For the symmetric case, assume that $f^{\prime}\left(s_{1}\right)=0$ for every $s_{1} \in[0, B)$. If an $\hat{s} \in(0, B) \times(0, B)$ exists so that the allocation $A[\hat{s}]$ of the $t$-player is quasi-bundling, $g^{\prime}>0$ is continuous in the point $\hat{s}_{2}$, and $f>0$ is continuous in the point $\hat{s}_{1}$, then the mechanism is a relaxed affine minimizer (with $\gamma_{1}=\infty$ ).

Proof. Assume w.l.o.g. that the conditions of the first statement of the lemma hold. We use an analogous claim as in the previous lemma:

Claim 2. Assume that $f^{\prime}>0$ is continuous as univariate function in the points $s_{1}$ and $s_{1}+\delta$, and $g>0$ is continuous in the points $s_{2}$, and $s_{2}-\delta$, furthermore the allocation of the $t$-player is quasi-bundling in a $2 \delta$-neighborhood of $s=\left(s_{1}, s_{2}\right)$. then $f^{\prime}\left(s_{1}+\delta\right)-f^{\prime}\left(s_{1}\right)=g\left(s_{2}\right)-g\left(s_{2}-\delta\right)$.

Proof. (Figure 7(b)) Assume for contradiction first that $f^{\prime}\left(s_{1}+\delta\right)-f^{\prime}\left(s_{1}\right)>g\left(s_{2}\right)-g\left(s_{2}-\delta\right)$. Since $f^{\prime}$ is continuous in $s_{1}+\delta$, it even holds that $f^{\prime}\left(s_{1}+\delta^{\prime}\right)-f^{\prime}\left(s_{1}\right)>g\left(s_{2}\right)-g\left(s_{2}-\delta\right)$ for some $\delta^{\prime}<\delta$.

Let $s^{\prime}=\left(s_{1}+\delta^{\prime}, s_{2}-\delta\right)$. Now there exists a point $\hat{t}$ so that $\hat{t} \in R_{\emptyset}(s)$ but $\hat{t} \in R_{12}\left(s^{\prime}\right)$. In turn, for this bid $\hat{t}$ of the $t$-player the $s$-player receives both tasks 1,2 if he bids $s$, and he receives no task if he bids $s^{\prime}$. This contradicts WMON for the $s$-player because $s_{1}+s_{2}>s_{1}+s_{2}+\delta^{\prime}-\delta=$ $s_{1}^{\prime}+s_{2}^{\prime}$.

Assume now that $f^{\prime}\left(s_{1}+\delta\right)-f^{\prime}\left(s_{1}\right)<g\left(s_{2}\right)-g\left(s_{2}-\delta\right)$. Since $f^{\prime}$ is continuous in $s_{1}+\delta$, it even holds that $f^{\prime}\left(s_{1}+\delta^{\prime}\right)-f^{\prime}\left(s_{1}\right)<g\left(s_{2}\right)-g\left(s_{2}-\delta\right)$ for some $\delta^{\prime}>\delta$.

Let $s^{\prime}=\left(s_{1}+\delta^{\prime}, s_{2}-\delta\right)$. Now there exists a point $\hat{t}$ so that $\hat{t} \in R_{12}(s)$ but $\hat{t} \in R_{\emptyset}\left(s^{\prime}\right)$. In turn, for this bid $\hat{t}$ of the $t$-player the $s$-player receives no task if he bids $s$, and he receives both tasks if he bids $s^{\prime}$. This contradicts WMON for the $s$-player because $s_{1}+s_{2}<s_{1}+s_{2}+\delta^{\prime}-\delta=s_{1}^{\prime}+s_{2}^{\prime}$.

Since $g^{\prime} \equiv 0$, the allocation is quasi-bundling whenever $f^{\prime}(s)>0$ and $g(s)>0$. By fixing $\bar{s}_{1}$ we fix an $f^{\prime}>0$, because $f^{\prime}$ is continuous in $\bar{s}_{1}$ and therefore independent of $s_{2}$.

Like in the proof of Lemma 11, we can show that $g\left(s_{2}\right)=P_{12}\left(s_{2}\right)-P_{1}$ is a (truncated) linear function over $s_{2} \in(0, B)$. In turn, by fixing $\bar{s}_{2}$, and thus fixing a $g>0$, we obtain that $f^{\prime}=P_{1}\left(s_{1}\right)$ is truncated linear over $s_{1} \in(0, B)$ with the same derivative as $g$.

Assume finally, that $f^{\prime}\left(s_{1}\right)=0$ iff $s_{1} \leq D$, for some $D>0$, and $g\left(s_{2}\right)=\mu s_{2}+C$ for an $C>0$. Then for $\left(s_{1}+s_{2}\right) \in[0, D)$ the $P_{12}\left(s_{1}+s_{2}\right)$ is an arbitrary increasing function $P_{12}:[0, D) \rightarrow[0, C)$, because there exists no $s>0$ such that $s_{1}+s_{2}<D$ but $f^{\prime}\left(s_{1}\right)>0$ would hold, and $g$ would be defined (and similarly for $t_{1}+t_{2}<C$ ) so $P_{12}$ needs not be linear over this interval. These $s$ and $t$ values having sum of coordinates below the given constants $D$ and $C$, respectively, are the only exceptions where the mechanism is a 1-dimensional bundling mechanism and not an affine minimizer.

Lemma 13. Assume that an $\hat{s} \in(0, B) \times(0, B)$ exists so that $f^{\prime}>0$ and $f>0$ are defined and both are continuous in the point $\hat{s}_{1}$; and $g^{\prime}>0$ and $g>0$ are defined and both are continuous in the point $\hat{s}_{2}$. If the allocation $A[\hat{s}]$ of the t-player is quasi-flipping then the mechanism is an affine minimizer; if $A[\hat{s}]$ is quasi-bundling, then the mechanism is a relaxed affine minimizer. 
Proof. Assume first that the allocation $A[\bar{s}]$ is quasi-flipping, i.e., $0<f(\bar{s})<f^{\prime}(\bar{s})$ and $0<$ $g(\bar{s})<g^{\prime}(\bar{s})$. A claim analogous to Claim 1 says that $f$ and $g$ must have equal difference quotients:

Claim 3. Assume that $f>0$ is continuous as univariate function in the points $s_{1}$ and $s_{1}+\delta$, and $g>0$ is continuous in the points $s_{2}$, and $s_{2}+\delta$, furthermore the allocation of the $t$-player is quasi-flipping in a $2 \delta$-neighborhood of $s=\left(s_{1}, s_{2}\right)$. Then $f\left(s_{1}+\delta\right)-f\left(s_{1}\right)=g\left(s_{2}+\delta\right)-g\left(s_{2}\right)$.

Let us now fix $s_{1}=\bar{s}_{1}$. Since $f$ and $f^{\prime}$ are independent of $s_{2}$ for this fixed $\bar{s}_{1}$, Claims 1 and 3 can be applied, (as in the proof of Lemma 11), to prove that $g$ and $g^{\prime}$ are truncated linear functions with the same multiplicative constant $\mu$ that is at the same time the derivative of $f$ and $f^{\prime}$ in the point $\bar{s}_{1}$. (For the applicability of the claims notice that $A[s]$ remains quasi-flipping in some neighborhood of $\bar{s}$, since $f<f^{\prime}$ are continuous in $\bar{s}_{1}$, and in continuous points even independent of $s_{2}$, so $f<f^{\prime}$ must hold in a neighborhood.) Now, fixing $\bar{s}_{2}$ and switching the roles of $f$ and $g$ we obtain that $f$ and $f^{\prime}$ are also truncated linear functions with multiplicative constant $\mu$.

The allocation remains quasi-flipping (because $f<f^{\prime}$ ) as long as $f^{\prime}>0$ and $g^{\prime}>0$. At least one of $f$ or $g$ is defined for every $s \in[0, B) \times[0, B)$. Therefore, at least three of $f, g, f^{\prime}, g^{\prime}$ are always uniquely defined (by the respective truncated linear functions), and they determine the allocation of the $t$-player (up to tie-breaking), which determines the allocation of the $s$-player. The mechanism must be an affine minimizer.

Second, assume that $A[\bar{s}]$ is quasi-bundling. We can use Claim 2 to prove that $f, g, f^{\prime}$ and $g^{\prime}$ are truncated linear with the same multiplicative constant $\mu$ on their whole domain, and $A[s]$ remains quasi-bundling as long as $f^{\prime}>0$ or $g^{\prime}>0$ holds. This determines the allocation as an affine minimizer if $f^{\prime}>0$ or $g^{\prime}>0$, because then at least three of $f, g, f^{\prime}$ or $g^{\prime}$ are defined. This is not the case if $f^{\prime}=g^{\prime}=0$, i.e. when the allocation is fully bundling. $P_{12}(x)$ is determined only if an $s=\left(s_{1}, s_{2}\right)$ with $x=s_{1}+s_{2}$ exists so that $f^{\prime}\left(s_{1}\right)>0$ or $g^{\prime}\left(s_{2}\right)>0$. If (for $x<D$ for a given constant $D>0)$ no such $s$ exists, then $P_{12}(x)$ is some arbitrary increasing function $P_{12}:[0, D) \rightarrow[0, C)$ and the mechanism becomes 1-dimensional. $C$ is here a constant playing the same role for the $t$ as $D$ for $s$. All in all, the mechanism is a relaxed affine minimizer.

CASE A. $\exists \bar{s}=\left(\bar{s}_{1}, \bar{s}_{2}\right) \in(0, B) \times(0, B)$ so that $f^{\prime}(\bar{s})>0, f^{\prime}$ as a function of $s_{1}$ is continuous in the point $\bar{s}_{1}$, and the allocation of the $t$-player is quasi-flipping or quasi-bundling, (OR the analogous case holds for $g^{\prime}$ );

Lemma 14. In CASE A. the conditions of at least one of Lemmas 11, 12 or 13 hold, and so the mechanism is a (relaxed) affine minimizer over positive valuations $s$ and $t$.

Proof.

Case 1. in $\bar{s}$ the allocation of the t-player is quasi-flipping

Since the allocation is quasi-flipping, it follows that $f^{\prime}>0$ and $g^{\prime}>0$. Moreover at least one of $f$ or $g$ is defined in $\bar{s}$, in which case $f<f^{\prime}$ and $g<g^{\prime}$, respectively.

\section{Case $1.1 f$ is defined for $\bar{s}$ in the allocation of the $t$-player}

We show first that we can assume w.l.o.g. that all of $f^{\prime}, f$ and $g^{\prime}$ are continuous as univariate functions in the points $\bar{s}_{1}$, and $\bar{s}_{2}$, respectively, otherwise we could slightly decrease $\bar{s}_{1}$, and after that, slightly increase $\bar{s}_{2}$, so that the continuity of all these critical value functions holds, and the allocation is still quasi-flipping:

If $f^{\prime}$ and $f$ are both continuous in $\bar{s}_{1}$, then set $\hat{s}_{1}=\bar{s}_{1}$. If $f^{\prime}$ and $f$ are not both continuous in $\bar{s}_{1}$, then we can reduce $\bar{s}_{1}$ to $\hat{s}_{1}=\bar{s}_{1}-\delta$ for an arbitrarily small value $\delta$ so that $f^{\prime}$ remains nearly the same and continuous, and $f$ is continuous (if still defined) in $\hat{s}_{1}$. Since $f^{\prime}$ does not change much, and $f$ cannot increase, the allocation is still quasi-flipping in $\left(\hat{s}_{1}, \bar{s}_{2}\right)$.

We need to show that $f$ is still defined in $\left(\hat{s}_{1}, \bar{s}_{2}\right)$. For this purpose, consider a point $\hat{t}=$ $\left(f^{\prime}(\bar{s})-\epsilon, g^{\prime}(\bar{s})-2 \epsilon\right)$. Since the allocation is quasi-flipping in $\bar{s}$, it holds that $\hat{t} \in R_{2}(\bar{s})$, but 
if $f^{\prime}\left(\hat{s}_{1}\right)>\bar{s}_{1}-\epsilon$, then it would get into $R_{1}$ or into $R_{12}$, unless the allocation is still quasiflipping with a well-defined $f$. Therefore, for this $\hat{t}, \bar{s} \in R_{1}^{s}$, but $\left(\hat{s}_{1}, \bar{s}_{2}\right) \in R_{2}^{s} \cup R_{\emptyset}^{s}$ would hold, contradicting WMON.

Now, given that $f^{\prime}$ and $f$ are continuous in $\bar{s}_{1}$, we can set $\hat{s}_{2} \geq \bar{s}_{2}$, so that $g^{\prime}$ is continuous, and if $f>0$ then $g$ is (defined and) continuous in $\hat{s}_{2} ; f$ and $f^{\prime}$ do not change, since in $\bar{s}_{1}$ both are independent of $s_{2}$. Therefore, the allocation in $\hat{s}$ is quasi-flipping.

Case 1.1.1 $f(\hat{s})>0$

By Lemma 13 , in this case the mechanism is an affine minimizer.

Case 1.1.2 $f(\hat{s})=0$

Case 1.1.2.1 both $f$ and $g$ are either zero or undefined for every $\left(s_{1}, s_{2}\right)$

By Lemma 11, in this case the mechanism is an affine minimizer.

Case 1.1.2.2 $\exists s$ so that $f(s)>0$ or $g(s)>0$

We show that even a point $s^{\prime}$ exists where the allocation is quasi-flipping, and each of $f, f^{\prime}, g, g^{\prime}$ are positive and continuous in $s^{\prime}$ in as univariate functions. Then the allocation is an affine minimizer by Lemma 13 .

Recall that $f^{\prime}$, and $f=0$ are continuous in the point $\hat{s}_{1}$, and $g^{\prime}$ is continuous in the point $\hat{s}_{2}$, moreover the allocation $A[\hat{s}]$ is quasi-flipping. Since by assumption an $s=\left(s_{1}, s_{2}\right)$ exists so that $f(s)>0$ or $g(s)>0$, this must be the case for every $s^{\prime}$ with $s_{1}^{\prime} \geq s_{1}$ and $s_{2}^{\prime} \geq s_{2}$, by Lemma 9. Therefore we can assume w.l.o.g. that $s_{1}^{\prime}>\hat{s}_{1}$ and $s_{2}^{\prime}>\hat{s}_{2}$. But then, by the same lemma $f$ is defined in $s^{\prime}$ (since $f$ is defined in $\hat{s}$ ), and it cannot be that $f\left(s^{\prime}\right)=0$, because then $g$ would be undefined (or zero) in $s^{\prime}$ as well. So $f\left(s^{\prime}\right)>0$ holds. Since $f\left(s^{\prime}\right), f^{\prime}\left(s^{\prime}\right)$ and $g^{\prime}\left(s^{\prime}\right)$ are all positive, $g\left(s^{\prime}\right)$ must be defined and $g\left(s^{\prime}\right)>0$ must hold, too. Finally, we assume w.l.o.g. that $f^{\prime}$ and $f$ are continuous in $s_{1}^{\prime}$ and $g^{\prime}$ and $g$ are continuous in the point $s_{2}^{\prime}$, because the (univariate) continuity holds in almost every $s_{1}^{\prime}$ and $s_{2}^{\prime}$.

We need to show that the allocation is quasi-flipping in $s^{\prime}$. We first consider the allocation in the point $\left(\hat{s}_{1}, s_{2}^{\prime}\right)$. Since for $\hat{s}_{1}$ the $f$ and $f^{\prime}$ are independent of $s_{2}$, they do not change as $\hat{s}_{2}$ gets changed to $s_{2}^{\prime}$, and the allocation remains quasi flipping. Now let us increase $\hat{s}_{1}$ to $s_{1}^{\prime}$. We prove that $A\left[s^{\prime}\right]$ is still quasi-flipping, so that Lemma 13 can be applied with $s^{\prime}$ playing the role of $\hat{s}$.

Assume for contradiction that $A\left[s^{\prime}\right]$ is crossing or quasi-bundling. Since in $s^{\prime}$ each of $f, f^{\prime}, g, g^{\prime}$ are defined and continuous, we can decrease $s_{2}^{\prime}$ to $s_{2}^{\prime}-\delta$, by an arbitrarily small $\delta$, the $f$ and $f^{\prime}$ do not change, and the allocation is still crossing or quasi-bundling. Now there is a point $\hat{t}=\left(0, g^{\prime}\left(s_{2}^{\prime}\right)-\epsilon\right)$ so that $\hat{t} \in R_{1}\left(\hat{s}_{1}, s_{2}^{\prime}\right)$ but $\hat{t} \in R_{12}\left(s_{1}^{\prime}, s_{2}^{\prime}-\delta\right)$. This contradicts WMON for the $s$-player for this $\hat{t}$, because for $\left(\hat{s}_{1}, s_{2}^{\prime}\right)$ he gets task 2 , but with bid $\left(s_{1}^{\prime}, s_{2}^{\prime}-\delta\right)$ he gets no task.

Case 1.2 $g$ is defined for $\bar{s}$ in the allocation of the $t$-player

There exists a $s_{2}^{*}>\bar{s}_{2}$ so that in this point $g^{\prime}$ and $g$ are both continuous as univariate functions. Note that $f^{\prime}\left(\bar{s}_{1}, s_{2}^{*}\right)=f^{\prime}(\bar{s})$ by Lemma 8 . We claim that in the point $\left(\bar{s}_{1}, s_{2}^{*}\right)$ the allocation of the $t$-player is quasi-flipping, so that we can use the same argument as in CASE 1.1.1, with $g$ and $g^{\prime}$ playing the roles of $f$ and $f^{\prime}$, respectively. This will prove that the mechanism is an affine minimizer.

Assume for contradiction that for $\left(\bar{s}_{1}, s_{2}^{*}\right)$ the allocation is crossing or quasi bundling. Notice that in this case $g\left(s_{2}^{*}\right) \geq g^{\prime}\left(s_{2}^{*}\right) \geq g^{\prime}\left(\bar{s}_{2}\right)>0$. The first inequality holds since the allocation became crossing or quasi-flipping; the second holds by the monotonicity of $g^{\prime}$.

Finally, we reduce $\bar{s}_{1}$ to $s_{1}^{*}=\bar{s}_{1}-\delta$, so that $f^{\prime}\left(s_{1}^{*}\right) \approx f^{\prime}\left(\bar{s}_{1}\right)$. Let $s^{*}=\left(s_{1}^{*}, s_{2}^{*}\right)$. Since $g$ and $g^{\prime}$ are independent of $s_{1}$, and $f^{\prime}\left(s_{1}^{*}\right) \approx f^{\prime}\left(\bar{s}_{1}\right)$, the allocation of the $t$-player is nearly the same for $s^{*}$ as for $\left(\bar{s}_{1}, s_{2}^{*}\right)$, and in particular $g\left(s^{*}\right) \geq g^{\prime}(\bar{s})$ still holds.

Now, using $g\left(s^{*}\right) \geq g^{\prime}(\bar{s})$, and $f^{\prime}\left(s^{*}\right) \approx f^{\prime}(\bar{s})$, it can be shown that now there exists a $t$ point so that $t \in R_{2}(\bar{s})$ but $t \in R_{12}\left(s^{*}\right)$. Consequently, for this $t$ point, $\bar{s} \in R_{1}^{s}(t)$ and $s^{*} \in R_{\emptyset}^{s}(t)$, contradicting monotonicity, since $s_{1}^{*}=\bar{s}_{1}-\delta$. 
Case 2. in $\bar{s}$ the allocation $A[\bar{s}]$ of the $t$-player is quasi-bundling

Since $f^{\prime}>0$, and the allocation is quasi-bundling, $g(\bar{s})$ is defined and positive.

Case $2.1 g^{\prime}(\bar{s})>0$

Since $g^{\prime}>0$, and the allocation is quasi-bundling, $f(\bar{s})$ is also defined, and $f^{\prime}<f$. If $f$ is continuous in the point $\bar{s}_{1}$ then set $\hat{s}_{1}=\bar{s}_{1}$. Otherwise set $\hat{s}_{1}=\bar{s}_{1}+\delta$ for some small $\delta>0$, so that both $f$ and $f^{\prime}$ are continuous in the point $\hat{s}_{1}$, and therefore independent of $s_{2}$. If $\delta$ is small enough then the allocation remains quasi-bundling, because $f$ is increasing in $s_{1}$ and $f^{\prime}$ is continuous in the point $\bar{s}_{1}$. Finally, if $g^{\prime}$ or $g$ are both continuous in the point $\bar{s}_{2}$, then set $\hat{s}_{2}=\bar{s}_{2}$. Otherwise set $\hat{s}_{2}=\bar{s}_{2}+\delta$ so that $g^{\prime}$ and $g$ are continuous (and positive) in the point $\hat{s}_{2}$. Since $f^{\prime}<f$ still holds, the allocation is quasi-bundling, and by Lemma 13 it is a relaxed affine minimizer.

Case $2.2 g^{\prime}(\bar{s})=0$

Case 2.2.1. $\exists s_{2}$ so that $g^{\prime}\left(s_{2}\right)>0$

Our goal is to increase $\bar{s}_{1}$ and $\bar{s}_{2}$ so that $g^{\prime}$ becomes positive, and CASE 1.2.1 applies.

We pick an $\hat{t}=\left(f^{\prime}\left(\bar{s}_{1}\right)+\epsilon, 0\right) \in R_{12}(\bar{s})$. This is possible, since $A[\bar{s}]$ is quasi-bundling. It holds then the $s$-player gets no task with bids $\bar{s}$ and $\hat{t}$. We increase $\bar{s}_{1}$ to $s_{1}^{*}$ so that $f^{\prime}\left(s_{1}^{*}\right)<f^{\prime}\left(\bar{s}_{1}\right)+\epsilon$, and $f^{\prime}$ is continuous as univariate function in $s_{1}^{*}$. This is possible since $f^{\prime}$ is continuous in $\bar{s}_{1}$. We also increase $\bar{s}_{2}$ to $s_{2}^{*}$, so that $g^{\prime}$ is strictly positive in the point $s_{2}^{*}$. We claim, that in $s^{*}=\left(s_{1}^{*}, s_{2}^{*}\right)$ the allocation $A\left[s^{*}\right]$ of the $t$-player is quasi-bundling, and therefore with $s^{*}$ in the role of $\bar{s}$, the proof can be completed as in CASE 1.2.1.

For $\hat{t}$ the $s$-player gets no task with bid $\bar{s}$ (see above), thus by WMON he also gets no task with bid $s^{*}$, since $\bar{s}<s^{*}$. In turn, the $t$-player gets both tasks with bids $s^{*}$ and $\hat{t}$. Given that $f^{\prime}\left(s_{1}^{*}\right)<f^{\prime}\left(\bar{s}_{1}\right)+\epsilon=\hat{t}_{1}$, this proves that the allocation $A\left[s^{*}\right]$ of the $t$-player is quasi-bundling.

Case 2.2.2. $g^{\prime}\left(s_{2}\right)=0$ for all $s_{2} \in[0, B)$

If $g$ is continuous in the point $\bar{s}_{2}$, then we can apply Lemma 12 with $\hat{s}=\bar{s}$.

If $g$ has a jump in $\bar{s}_{2}$, then let $\hat{s}_{1}=\bar{s}_{1}$, and $\hat{s}_{2}=\bar{s}_{2}+\delta$ so that $g$ is continuous in the point $\hat{s}_{2}$. Then $g$ is defined in $\hat{s}$, and $g\left(\hat{s}_{2}\right)>g\left(\bar{s}_{2}\right)>0$ by Lemma 9 . Since $g^{\prime}\left(\hat{s}_{2}\right)=0$ by the case assumption, $g(\hat{s})>g^{\prime}(\hat{s})$, so the allocation is quasi-bundling, and again we can apply Lemma 12 with $\hat{s}=\bar{s}$.

CASE B. CASE A. does not hold, but $\exists \bar{s}=\left(\bar{s}_{1}, \bar{s}_{2}\right) \in(0, B) \times(0, B)$ so that $f^{\prime}(\bar{s})=0$, $f^{\prime}$ as a function of $s_{1}$ is continuous in the point $\bar{s}_{1}$, and the allocation of the $t$-player is quasi-bundling, (OR the analogous case holds for $g^{\prime}$ );

Claim 4. In CASE B $f^{\prime}(s) \equiv 0$ and $g^{\prime}(s) \equiv 0$ for every $s \in[0, B) \times[0, B)$.

Proof. In $\bar{s}$ the $f^{\prime}$ is independent of $s_{2}$, whereas $g^{\prime}$ is increasing in $s_{2}$. Assume first for contradiction that $g^{\prime}>0$ for some $s_{2}$, and take an $s_{2}^{*}>\bar{s}_{2}$ point where $g^{\prime}$ is continuous, and strictly positive.

Since CASE A does not hold (for $g^{\prime}$ ), in $\left(\bar{s}_{1}, s_{2}^{*}\right)$ the allocation of the $t$-player must be crossing, while $f^{\prime}=0$ still holds.

For $\bar{s}$ the allocation was bundling, so there is a point $t=(\epsilon, \epsilon)$ that is in $R_{12}(\bar{s})$ (because of the bundling alloc.) and in $R_{2}\left(\bar{s}_{1}+\delta, s_{2}^{*}\right)$ (because of the crossing alloc with $g^{\prime}>0$, and because $f^{\prime}$ is continuous and zero in $\bar{s}_{1}$, it can be continuous and arbitrarily small or zero in some $\bar{s}_{1}+\delta$ ). For this $t$ in the allocation of the $s$-player $\bar{s} \in R_{\emptyset}^{s}$ but $\left(\bar{s}_{1}+\delta, s_{2}^{*}\right) \in R_{1}^{s}$, contradicting WMON. We obtained that $g^{\prime} \equiv 0$. By symmetric argument, using the existence of a bundling allocation in $\bar{s}$, and continuous $g^{\prime}$ there, we obtain $f^{\prime} \equiv 0$.

Corollary 3. Over $t \in(0, \infty) \times(0, \infty)$ and $s \in[0, B) \times[0, B)$ the mechanism is 1-dimensional mechanism bundling the two tasks; for $t_{1}=0$ and/or $t_{2}=0$ the allocation of the s-player can be non-bundling. 
CASE C. for every $s \in(0, B) \times(0, B)$ point where either $f^{\prime}$ or $g^{\prime}$ is continuous (as univariate functions), the allocation of the $t$-player is crossing

It is easy to show that in every point $s$ where $f$ or $g$ as univariate function is continuous in the point $s_{1}$, resp $s_{2}$, the allocation must be crossing (otherwise we cound change $s$ by a small value so that both $f$ and $f^{\prime}$ (or $g$ and $g^{\prime}$ ) would be continuous and the allocation would be still non-crossing). Thus, the allocation can be quasi-flipping or quasi-bundling, only in points where each of $f, g$ (when defined), and $f^{\prime}$ and $g^{\prime}$ have a jump discontinuity as univariate functions. In this case the allocation function corresponds to a relaxed task-independent mechanism, or 1-dimensional mechanism such that the $t$-player never gets task 1 or he never gets task 2 .

\section{B Mechanisms for 2 tasks and 2 machines with submodular valuations}

In this section we relax the assumption of additivity of the valuation of the $t$-player in different ways, and show in Theorem 5 that for all these extensions of the additive domain the only remaining truthful mechanisms are relaxed affine minimizers (or constant mechanisms), and 1dimensional mechanisms. This result holds for (i) arbitrary (normalized, monotone) valuations, (ii) submodular or subadditive valuations? (iii) $\epsilon$-additive valuations (see $V_{\epsilon}$ below), and (iv) for valuations that are submodular and $\epsilon$-additive. In essence, we characterize mechanisms for two tasks and two players in case (i), where the $t$-player has arbitrary monotone valuation $\left(t_{1}, t_{2}, t_{12}\right)$, and the $s$-player has additive valuation $\left(s_{1}, s_{2}, s_{1}+s_{2}\right)$ with $s_{1}$ and $s_{2}$ both bounded by an arbitrarily large fixed value $B$. We use the characterization for two additive players from Section $\mathrm{A}$, and show that relaxed task-independent mechanisms are not extendable onto the nonadditive domain, unless they are task-independent affine minimizers. Along the proof we argue that our lemmas carry over straightforward to cases (ii), (iii) and (iv) as well. In particular, task-independent mechanisms cannot be extended even to an arbitrarily 'narrow' superset $V_{\epsilon}$ of the (2-dimensional) additive domain in 3-dimensions.

\section{B.1 Basic concepts and notation}

For simplicity of presentation we consider any bid of either of the players as a vector of three entries. We summarize the notation for the considered domains of valuations (cost functions) as follows:

Definition 16. $V \subset \mathbb{R}_{\geq 0}^{3}$ is the $3 \mathrm{D}$ set of all monotone valuations:

$$
V=\left\{\left(x_{1}, x_{2}, x_{12}\right) \in \mathbb{R}_{\geq 0}^{3} \mid x_{12} \geq x_{1} \geq 0, x_{12} \geq x_{2} \geq 0\right\} ;
$$

$V_{+} \subset V$ is the additive plane:

$$
V_{+}=\left\{\left(x_{1}, x_{2}, x_{12}\right) \in \mathbb{R}_{\geq 0}^{3} \mid x_{1}, x_{2} \geq 0, x_{12}=x_{1}+x_{2}\right\}
$$

$V_{+, B} \subset V_{+}$is the additive plane, restricted to $[0, B) \times[0, B)$ for some arbitrarily large fixed $B$

$$
V_{+, B}=\left\{\left(x_{1}, x_{2}, x_{12}\right) \in \mathbb{R}_{\geq 0}^{3} \mid 0 \leq x_{1}, x_{2}<B, x_{12}=x_{1}+x_{2}\right\} ;
$$

$V_{\epsilon} \subset V$ is the $\epsilon$-neighborhood 10 of the additive plane for some arbitrary fixed $\epsilon>0$ :

$$
V_{\epsilon}=\left\{\left(x_{1}, x_{2}, x_{12}\right) \in V \mid x_{12} \in\left(x_{1}+x_{2}-\epsilon, x_{1}+x_{2}+\epsilon\right)\right\} ;
$$

\footnotetext{
${ }^{9}$ for two tasks subadditive and submodular valuations are the same

${ }^{10} \mathrm{We}$ are quite free to use any reasonable definition of such a neighborhood, e.g., we could have chosen $x_{12} \in$ $\left((1-\epsilon)\left(x_{1}+x_{2}\right),(1+\epsilon)\left(x_{1}+x_{2}\right)\right)$, or even the intersection of the latter with the current (additive) neighborhood. The characterization is not very sensitive to this definition.
} 
$V_{\text {submod }} \subset V$ is the 3D set of all submodular valuations; for two tasks this is equivalent with subadditivity:

$$
V_{\text {submod }}=\left\{\left(x_{1}, x_{2}, x_{12}\right) \in V \mid x_{12} \leq x_{1}+x_{2}\right\} .
$$

Let $V^{*} \in\left\{V, V_{\epsilon}, V_{\text {submod }}, V_{\text {submod }} \cap V_{\epsilon}\right\}$, and let $(A, \hat{P})$ denote the allocation and payment functions of a truthful mechanism on $V^{*} \times V_{+, B}$. For the valuations $t=\left(t_{1}, t_{2}, t_{12}\right)$ and $s=$ $\left(s_{1}, s_{2}, s_{12}\right)$ taken from some given domains $t \in V^{*}$ and $s \in V_{+, B}$, the allocation is $A(t, s)=$ $\left(\alpha_{t}, \alpha_{s}\right)$, where $\alpha_{t}, \alpha_{s} \in\{12,1,2, \emptyset\}$. Since both tasks are always allocated, $\alpha_{t}$ determines $\alpha_{s}=$ $\bar{\alpha}_{t}$, and for simplicity we often identify $A(t, s)$ with the allocation $\alpha_{t}$ to the $t$-player.

For given $s \in V_{+, B}$, and $\alpha \in\{12,1,2, \emptyset\}$, the interior (w.r.t. $V^{*}$ ) of the set of all $t \in V^{*}$ where the $t$-player is allocated $\alpha$ is denoted by $R_{\alpha}(s) \subset V^{*}$. We omit $(s)$ from the notation if $s$ is clear from the context. $R_{\alpha}(s)$ is an open set in $V^{*}$. Similarly, for given $t \in V^{*}$, the interior (w.r.t. $V_{+, B}$ ) of the set of all $s \in V_{+, B}$ where the $s$-player is allocated $\alpha$ is denoted by $R_{\alpha}^{s}(t) \subset V_{+, B}$.

We will assume regarding allocations of the $t$-player that $R_{\emptyset}(s)$ is nonempty for every $s$. This assumption is without loss of generality for allocations $A$ with finite approximation ratio of the makespan. Since $R_{\emptyset} \neq \emptyset$, we can assume w.l.o.g. that the payment function $\hat{P}$ is normalized, that is, $\hat{P}_{\emptyset}(s)=0$ for every $s$.

The payments to the $t$-player for taking the tasks 12,1 , and 2 respectively, depending on the bid $s$ are denoted by $\hat{P}_{12}(s), \hat{P}_{1}(s), \hat{P}_{2}(s)$. We omit the argument $s$ if it is clear from the context. The payments to the $s$-player depending on the bid $t$ are denoted by $\hat{P}_{12}^{s}(t), \hat{P}_{1}^{s}(t), \hat{P}_{2}^{s}(t)$.

Observe that a truthful mechanism $(A, \hat{P})$ over $V^{*} \times V_{+, B}$ is a truthful mechanism when restricted to $V_{+} \times V_{+, B}$. We denote this restricted mechanism by $\left(\left.A\right|_{V_{+} \times V_{+, B}},\left.\hat{P}\right|_{V_{+} \times V_{+, B}}\right)$.

We introduce the types of WMON allocations that can occur on $V^{*} \times V_{+, B}$.

\section{B.1.1 Relaxed affine minimizers}

Definition 17. An allocation $A$ is an affine minimizer, if there exist positive constants per player $\mu_{t}$ and $\mu_{s}$, and constants $\gamma_{\alpha} \in \mathbb{R} \cup\{-\infty, \infty\}$ per allocation (say, here $\alpha=\alpha_{t}$ ), so that for every input $(t, s)$ the allocation $A(t, s)$ minimizes over

$$
\mu_{t} \cdot t_{12}+\gamma_{12}, \quad \mu_{t} \cdot t_{1}+\mu_{s} \cdot s_{2}+\gamma_{1}, \quad \mu_{s} \cdot s_{1}+\mu_{t} \cdot t_{2}+\gamma_{2}, \quad \mu_{s}\left(s_{1}+s_{2}\right)+\gamma_{\emptyset} .
$$

Definition 18. An allocation $A$ is a relaxed affine minimizer, if there exist positive constants per player $\mu_{t}$ and $\mu_{s}$, and constants $\gamma_{\alpha}$ per allocation $\alpha$ (of the $t$-player), furthermore an arbitrary increasing function $\xi:\left[0, \min \left(\gamma_{1}, \gamma_{2}\right)-\gamma_{\emptyset}\right) \rightarrow[0, \infty)$ (if the interval $\left[0, \min \left(\gamma_{1}, \gamma_{2}\right)-\gamma_{\emptyset}\right)$ is nonempty) with $\min \left(\gamma_{1}, \gamma_{2}\right)-\gamma_{12}=\xi\left(\min \left(\gamma_{1}, \gamma_{2}\right)-\gamma_{\emptyset}\right)$, so that for every input $(t, s)$

a) if $\mu_{s} \cdot\left(s_{1}+s_{2}\right) \geq \min \left(\gamma_{1}, \gamma_{2}\right)-\gamma_{\emptyset}$, the allocation $A(t, s)$ is that of an affine minimizer with the given constants

b) if $\mu_{s} \cdot\left(s_{1}+s_{2}\right) \leq \min \left(\gamma_{1}, \gamma_{2}\right)-\gamma_{\emptyset}$, then if $\mu_{t} \cdot t_{12}>\xi\left(\mu_{s}\left(s_{1}+s_{2}\right)\right)$ then the allocation for the $t$-player is $\emptyset$ and if $\mu_{t} \cdot t_{12}<\xi\left(\mu_{s} \cdot\left(s_{1}+s_{2}\right)\right)$ then it is 12 .

\section{B.1.2 Constant mechanisms}

In a constant mechanism the allocation is independent of the bids of at least one of the players. This property can also be interpreted as being an affine minimizer with multiplicative constant $\mu=0$ for this player.

\section{B.1.3 1-dimensional mechanisms}

In a 1-dimensional mechanism only at most two possible allocations can occur. If the two occuring allocations (for the $t$-player) are $\emptyset$ and 12 , we call the mechanism bundling mechanism. 
Definition 19. In a bundling mechanism only the allocations $\emptyset$ and 12 can occur. There is an arbitrary, increasing function $\xi:[0, B) \rightarrow[0, \infty)$ so that if $t_{12}>\xi\left(s_{1}+s_{2}\right)$ then the $t$-player gets $\emptyset$, and if $t_{12}<\xi\left(s_{1}+s_{2}\right)$ then the $t$-player gets 12 .

If $\xi$ has a jump discontinuity in some point $s_{1}+s_{2}$ then the critical value for the $t$-player to get the tasks may depend on the concrete $\left(s_{1}, s_{2}\right)$ with the given fixed sum, as long as it is between $\xi\left(\left(s_{1}+s_{2}\right)^{-}\right)$and $\xi\left(\left(s_{1}+s_{2}\right)^{+}\right)$.

In the other cases the only occuring allocations are $\emptyset$ and 1 or $\emptyset$ and 2 , respectively. The mechanism is in this case identical with a degenerate task-independent mechanism (see also Section A where task 2 (resp. task 1) is always received by the $s$-player. Such mechanisms can also be extended to $V \times V_{+B}$. Notice however, that all 1-dimensional mechanisms have arbitrarily high approximation ratio if we set the fixed bound $B$ high enough.

\section{B.2 The main result}

Theorem 5. Every WMON allocation for two tasks and two players with bids $t \in V_{\epsilon}$ and $s \in V_{+, B}$, where both tasks are always allocated, and $R_{\emptyset}(s) \neq \emptyset$ for every $s$, is one of these three types: (1) relaxed affine minimizer, (2) one-dimensional mechanism, or (3) constant mechanism. The same characterization holds for WMON allocations over $V^{*} \times V_{+, B}$, for $V^{*} \in$ $\left\{V, V_{\text {submod }}, V_{\epsilon}, V_{\text {submod }} \cap V_{\epsilon}\right\}$.

Remark. (a) We note that for $t \in V$ or $t \in V_{\text {submod }}$, Theorem 5 has a shorter, direct proof not using the characterization for additive players of Section A. However, to the best of our knowledge this direct proof does not carry over to the almost-additive domain $V_{\epsilon}$ for the $t$-player. (b) Arguments analogous to the following proof show that the same characterization holds, e.g., for player valuations $(t, s)$ in $V \times V$, or $V_{\text {submod }} \times V_{\text {submod }}$, or $V \times V_{+}$. Since this is not the central topic of this paper, and similar characterizations have been known, we do not consider these cases here: our primary goal is to show that even if the $s$-player remains additive and even bounded, and the $t$-player is almost additive, the 'disturbing' task-independent allocations disappear.

\section{B.3 Proof of Theorem 5}

\section{B.3.1 Virtual payments}

Let $(A, \hat{P})$ be a truthful mechanism for $(t, s)$ over $V^{*} \times V_{+, B}$, for $V^{*} \in\left\{V, V_{\text {submod }}, V_{\epsilon}, V_{\text {submod }} \cap\right.$ $\left.V_{\epsilon}\right\}$. We will use virtual payments for the $t$-player defined as follows.

Definition 20. For every truthful mechanism $(A, \hat{P})$ over $(t, s) \in V^{*} \times V_{+, B}$, or over $(t, s) \in$ $V_{+} \times V_{+, B}$, we define the virtual payments iteratively. Let $s$ be arbitrary fixed valuation from $V_{+, B}$. The payments of the $t$-player occuring in this definition are all defined for this given $s$.

$$
\begin{gathered}
P_{\emptyset}=0, \\
P_{1}=\max \left\{\hat{P}_{1}, 0\right\}, \quad P_{2}=\max \left\{\hat{P}_{2}, 0\right\}, \\
P_{12}=\max \left\{\hat{P}_{12}, P_{1}, P_{2}\right\} .
\end{gathered}
$$

Definition 21. We call a payment function $P$ monotone, if for every $s$, and sets of tasks $\beta \subset \alpha$, it holds that $P_{\beta}(s) \leq P_{\alpha}(s)$.

Claim 5. The virtual payments are normalized, monotone and $(A, P)$ is a truthful mechanism.

Proof. The virtual payment fuction $P$ is obviously normalized and monotone. We show that $P$ is truthful for the $t$-player for arbitrary $s$. We claim first that $P_{\alpha}(s)=\hat{P}_{\alpha}(s)$, whenever $R_{\alpha}(s)$ is nonempty. We show this for $\alpha=12$, the cases $\alpha=1$ and $\alpha=2$ are similar. Assume for 
contradiction that $t \in R_{\alpha}(s)$, but $P_{12} \neq \hat{P}_{12}$. In this case either $P_{12}=\hat{P}_{1}$, or $P_{12}=\hat{P}_{2}$, or $P_{12}=0=\hat{P}_{\emptyset}$, so the allocation $\alpha=1$, or $\alpha=2$, or $\alpha=\emptyset$, respectively, is at least as profitable as the more costly allocation $\alpha=12$, so $t$ must be in the closure of some other region and cannot be in the open interior $R_{12}$, a contradiction.

In order to prove the truthfulness of $P$, we need to prove that the given allocation $A(t)$ maximizes the profit $P-t$ for every $t \in V^{*}$ (resp. for every $t \in V_{+}$). For every fixed $t$ it holds that $t \in \bar{R}_{\alpha}$ for some $\alpha$ with $R_{\alpha} \neq \emptyset$. We show next that for this $\alpha, P_{\alpha}-t_{\alpha} \geq P_{\beta}-t_{\beta}$ for all $\beta \in\{12,1,2, \emptyset\}$.

If $R_{\beta} \neq \emptyset$, then, by the first paragraph, $P_{\alpha}=\hat{P}_{\alpha}$, and $P_{\beta}=\hat{P}_{\beta}$, so the inequality holds, because the $\hat{P}$ are truthful payments. Assume now, that $R_{\beta}=\emptyset$. Then $\beta \in\{1,2,12\}$, by the assumption $R_{\emptyset} \neq \emptyset$. If $\beta=1$, then $R_{1}=\emptyset$ and we claim that $P_{1}=0$ must hold. Otherwise $\hat{P}_{1}>0$, and the points $t=\left(t_{1}<\hat{P}_{1}, t_{2}=L, t_{12}=t_{1}+L\right)$ (for large enough $L$ ) are in $R_{1}$, (by truthfulness of $\hat{P}$ ) contradicting $R_{1}=\emptyset$. Now, it holds that $P_{\alpha}-t_{\alpha} \geq P_{\emptyset}=0 \geq 0-t_{1}=P_{1}-t_{1}$. Here the first inequality follows from the statement (that $P_{\alpha}=\hat{P}_{\alpha}$ and $P_{\emptyset}=\hat{P}_{\emptyset}$ ) about nonempty regions, and the truthfulness of $\hat{P}$. For $\beta=2$ the proof is analogous.

Assume finally that $\beta=12$. We claim that then $P_{12}=P_{1}$ or $P_{12}=P_{2}$. Otherwise $P_{12}=$ $\hat{P}_{12}>\max \left\{\hat{P}_{1}, \hat{P}_{2}, 0\right\}$, and by truthfulness of $\hat{P}$, for small enough $\epsilon>0$ the $t=(\epsilon, \epsilon, 2 \epsilon)$ must be a point in $R_{12}$, contradicting $R_{12}=\emptyset$. Assuming now w.l.o.g. $P_{12}=P_{1}$, it holds that $P_{\alpha}-t_{\alpha} \geq P_{1}-t_{1}=P_{12}-t_{1} \geq P_{12}-t_{12}$, where the first inequality was shown above, and the last inequality holds by monotonicity of the valuations. This concludes the proof.

In fact, for given WMON allocation function $A(t)$ as function of the bid $t$ of the $t$-player (in particular for the allocation function $A[s]$ for arbitrary fixed $s$ ), the virtual payments are the unique payments which are truthful, normalized and monotone, as we show next.

Claim 6. For every WMON allocation function $A(t)$ of the $t$-player (i.e., for fixed $s$ ) over $t \in V^{*}$ or over $t \in V_{+}, A(t)$ uniquely determines the virtual payments as the only truthful, normalized and monotone payments. Conversely, the virtual payments determine the allocation $A(t)$ up to tie-breaking.

Proof. For every $V^{*}$ it is well-known that the payments $P_{12}, P_{1}, P_{2}, P_{\emptyset}$ uniquely determine the truthful allocation up to tie-breaking, since the allocation maximizes $P_{\alpha}-t_{\alpha}$ over $\alpha \in$ $\{12,1,2, \emptyset\}$.

We prove the uniqueness of monotone, normalized, truthful payments for a given allocations function $A(t)$. By definition, $P_{\emptyset}=0$ must hold. We claim that, if $R_{1}=\emptyset$, then $P_{1}=0$ must be the case. Assume the contrary, then by monotonicity, $P_{1}>0$ must hold. However, if $P_{1}>0$, then the points $t=\left(t_{1}<P_{1}, t_{2}=L, t_{12}=t_{1}+L\right) \in V_{+} \subset V^{*}$ (for large enough $L$ ) are in $R_{1}$, (by truthfulness) contradicting $R_{1}=\emptyset$. If $R_{1} \neq \emptyset$, then $P_{1}=\sup \left\{t_{1} \mid\left(t_{1}, L, t_{1}+L\right) \in R_{1}\right\}$ is uniquely determined by $A(t)$. (Note that the supremum cannot be infinite, otherwise $R_{\emptyset}=\emptyset$ would hold.) By symmetric argument, also $P_{2}$ is uniquely determined by $A(t)$.

Consider now $P_{12}$. We claim that, if $R_{12}=\emptyset$, then $P_{12}=\max \left\{P_{1}, P_{2}, 0\right\}$ must be the case (these are unique by the paragraph above). Assume the contrary, that $P_{12}>\max \left\{P_{1}, P_{2}, 0\right\}$. Then, for every small enough $\epsilon>0$ the $t=(\epsilon, \epsilon, 2 \epsilon) \in V_{+} \subset V^{*}$ must be a point in $R_{12}$, contradicting $R_{12}=\emptyset$. Finally, if $R_{12} \neq \emptyset$, then $(0,0,0) \in R_{12}$ and $R_{12}$ must have boundary points $t$ with at least one of $R_{\emptyset}$, or $R_{1}$ or $R_{2}$, given that $R_{\emptyset} \neq \emptyset$. If $R_{12}$ has a boundary with (say) $R_{1}$, then $P_{12}-P_{1}=\sup \left\{t_{2} \mid\left(0, t_{2}, t_{2}\right) \in R_{12}\right\}$, because this point (i.e., with the supremum $t_{2}$ value) must be on the boundary with $R_{1}$. If $R_{12}$ has no common boundary with $R_{1}$ or $R_{2}$, then $P_{12}=\sup \left\{t_{2} \mid\left(0, t_{2}, t_{2}\right) \in R_{12}\right\}$, because this point must be on the boundary with $R_{\emptyset}$. 


\section{B.3.2 One-to-one correspondence of allocation and virtual payments}

The virtual payments determine the allocation so that critical values for a bid getting/losing a task (for fixed other bids) are always non-negative ${ }^{11}$ Exploiting this, it simplifies the discussion that we can assume that the closure of each of the 4 allocation regions of the $t$-player is always nonempty (over $V^{*}$ or $V_{+}$) : if for some $t \in \mathbb{R}_{\geq 0}^{3}$ an allocation (say 12) does not contradict truthfulness for the virtual payments, then we assume that $t$ is in the closure of the respective allocation region (say $t \in \bar{R}_{12}$ ). For example, w.l.o.g. $(0,0,0) \in \bar{R}_{12}$, and $(0, L, L) \in \bar{R}_{1}$ for large enough $L$ always hold, because $P_{12}$ is the largest payment, and $P_{1}$ is nonnegative. ${ }^{12}$ In the proofs we always pick bids $t$ from the interior of some allocation region (here the allocation is uniquely determined), so the above assumption is without loss of generality.

Claim 6 has a crucial, and maybe surprising implication concerning a one-to-one relation between WMON allocations $A(t)$ over $t \in V^{*}$ and their respective restrictions to the additive plane $\left.A\right|_{V_{+}}$.

Lemma 15. For every $W M O N$ allocation function $A(t)$ over $t \in V^{*}$ (in particular for the allocation function $A[s]$ for arbitrary fixed $s$ ), and its restriction to the additive plane $A_{+}:=\left.A\right|_{V_{+}}$ hold that $A$ uniquely determines $A_{+}$, and conversely, up to tie-breaking $A_{+}$uniquely determines $A$ over $V^{*}$. Moreover the virtual payments $P_{12}, P_{1}, P_{2}, P_{\emptyset}$ are the same for $A$ as for $A_{+}$.

Proof. The first statement, that $A$ determines $\left.A\right|_{V_{+}}$, is obvious. Since the payments for $A$ are normalized, monotone, and truthful for $A_{+}$, by uniqueness these are the only possible virtual payments for $A_{+}$.

We show how the other implication (that $A_{+}$determines $A$ ) also follows from the uniqueness of the normalized and monotone payments. Suppose that there exist two different WMON allocation functions over $V^{*}, A^{\prime}(t)$ and $A^{\prime \prime}(t)$, such that $A_{+}(t)=\left.A^{\prime}\right|_{V_{+}}(t)=\left.A^{\prime \prime}\right|_{V_{+}}(t)$ for every $t \in V_{+}$. By Claim 6 there exist unique virtual payments $P^{\prime}=\left(P_{12}^{\prime}, P_{1}^{\prime}, P_{2}^{\prime}, P_{\emptyset}^{\prime},\right)$ and $P^{2}=\left(P_{12}^{\prime \prime}, P_{1}^{\prime \prime}, P_{2}^{\prime \prime}, P_{\emptyset}^{\prime \prime},\right)$, i.e., truthful, normalized and monotone payments for $A^{\prime}$ and $A^{\prime \prime}$, respectively. Obviously, both are truthful, normalized and monotone for the allocation function $A_{+}(t)$, when restricted to $V_{+}$. Since $A_{+}$has unique truthful, normalized and monotone payments, by Claim 6 , it must be the case that $P_{12}^{\prime}=P_{12}^{\prime \prime}, P_{1}^{\prime}=P_{1}^{\prime \prime}, P_{2}^{\prime}=P_{2}^{\prime \prime}$, and $P_{\emptyset}^{\prime}=P_{\emptyset}^{\prime \prime}$. This, in turn, implies by the same claim applied on $V^{*}$ that $A^{\prime} \equiv A^{\prime \prime}$ up to tie-breaking, which concludes the proof.

\section{B.3.3 Illustration}

We provide a geometric illustration to Lemma 15. For fixed $s$, the allocation function to the $t$-player $A\left(t_{1}, t_{2}, t_{12}\right)$ has a very simple geometric representation in $\mathbb{R}_{\geq 0}^{3}$.

Consider the (closures of the) four allocation regions of the $t$-player in $V: \bar{R}_{12}, \bar{R}_{1}, \bar{R}_{2}, \bar{R}_{\emptyset}$. By truthfulness, these regions are uniquely determined by the three payments as follows:

$$
\begin{gathered}
\bar{R}_{\emptyset}=\left\{t \in V \mid t_{1} \geq P_{1}, t_{2} \geq P_{2}, t_{12} \geq P_{12}\right\}, \\
\bar{R}_{1}=\left\{t \in V \mid t_{1} \leq P_{1}, t_{1} \leq t_{12}+P_{1}-P_{12}, t_{1} \leq t_{2}+P_{1}-P_{2}\right\}, \\
\bar{R}_{2}=\left\{t \in V \mid t_{2} \leq P_{2}, t_{2} \leq t_{12}+P_{2}-P_{12}, t_{2} \leq t_{1}+P_{2}-P_{1}\right\}, \\
\bar{R}_{12}=\left\{t \in V \mid t_{12} \leq P_{12}, t_{12} \leq t_{1}+P_{12}-P_{1}, t_{12} \leq t_{2}+P_{12}-P_{2}\right\} .
\end{gathered}
$$

The boundaries between pairs of these regions, if exist, must be subsets of the following planes, respectively:

$$
\left\{t \in V \mid t_{1}=P_{1}\right\} \quad\left(R_{1} \mid R_{\emptyset}\right)
$$

\footnotetext{
${ }^{11}$ In geometric terms, axis-parallel boundaries always have position at least 0 .

${ }^{12}$ Mind that the closure of a region $\bar{R}_{\alpha}$ defined this way is not the topological closure of the respective interior $R_{\alpha}$, e.g., if $R_{\alpha}$ is empty.
} 


$$
\begin{array}{cr}
\left\{t \in V \mid t_{2}=P_{2}\right\} & \left(R_{2} \mid R_{\emptyset}\right) \\
\left\{t \in V \mid t_{12}=P_{12}\right\} & \left(R_{12} \mid R_{\emptyset}\right) \\
\left\{t \in V \mid t_{12}-t_{1}=P_{12}-P_{1}\right\} & \left(R_{12} \mid R_{1}\right) \\
\left\{t \in V \mid t_{12}-t_{2}=P_{12}-P_{2}\right\} & \left(R_{12} \mid R_{2}\right) \\
\left\{t \in V \mid t_{1}-t_{2}=P_{1}-P_{2}\right\} & \left(R_{2} \mid R_{1}\right) .
\end{array}
$$

All these planes have the common intersection point $\left(P_{1}, P_{2}, P_{12}\right)$. By monotonicity of the virtual payments, this common point is in the domain $V$ of monotone valuations, and it is a boundary point of all four regions in $V$. Moreover these boundaries always exist in $V$ (not only their planes). Their positions determine the allocation and the payments and vice-versa. The four regions in $V \subset \mathbb{R}_{\geq 0}^{3}$, of a general WMON allocation have thus a rather simple and visible structure.

Let us now consider the additive plane $V_{+}$; this plane always intersects the first two boundaries $\left(R_{1} \mid R_{\emptyset}\right.$ and $\left.R_{2} \mid R_{\emptyset}\right)$, so that the allocation on the additive plane uniquely determines $P_{1}$ and $P_{2}$. Finally, always at least one of the third, fourth and fifth boundaries intersects (as boundary) the additive plane $V_{+}$, which determines $P_{12}$.

In particular, as can be seen from the proof of Lemma 15, the interior of a region $R_{\alpha}$ of the $t$-player is empty in $V_{+}$if and only if it is empty in $V$ (and consequently, if and only if it is empty in $V^{*}$, since $V_{+} \subset V^{*}$ ). This may seem surprising, since it could in principle have been the case that the additive plane $V_{+}$does not intersect some nonempty region $R_{\alpha} \subset V$.

\section{B.3.4 Extending the additive characterization to $V^{*} \times V_{+, B}$}

In what follows, we characterize all truthful mechanisms for two tasks and two bidders with valuations on the domains $V \times V_{+, B}, V_{\text {submod }} \times V_{+, B}, V_{\epsilon} \times V_{+, B}$, and $\left(V_{\epsilon} \cap V_{\text {submod }}\right) \times V_{+, B}$ based on the characterization on $V_{+} \times V_{+, B}$. According to Theorem 4 , every truthful mechanism on $V_{+} \times V_{+, B}$ is one of the following four types: (1) relaxed affine minimizer, (2) relaxed taskindependent mechanism, (3) 1-dimensional mechanism, (4) constant mechanism.

Let $A$ be some WMON allocation on $V^{*} \times V_{+, B}$ for $V^{*} \in\left\{V, V_{\text {submod }}, V_{\epsilon}, V_{\text {submod }} \cap V_{\epsilon}\right\}$. (these are the allocations to be characterized). Its restriction $\left.A\right|_{V_{+} \times V_{+, B}}$ to additive valuations of the $t$-player is a WMON allocation for additive valuations, and therefore is of one of the types (1) to (4) above. Let $\left.A\right|_{V_{+} \times V_{+, B}}$ be for instance a (relaxed) affine minimizer. Then, for every $s \in V_{+, B}$ the allocation and payments for the $t$-player on $t \in V_{+}$are those of this affine minimizer. By uniqueness (Lemma 15), they extend to $t \in V^{*}$ uniquely to be the allocation and payments of the same affine minimizer. This, in turn, means that for every fixed $t \in V^{*}$ the $s$-player on $s \in V_{+, B}$ has allocation and payments according to this affine minimizer. Altogether the mechanism on $V^{*} \times V_{+, B}$ is the same affine minimizer, with the same parameters, and same virtual payments as on $V_{+} \times V_{+, B}$. The argument is analogous for 1-dimensional mechanisms and for constant mechanisms. On the other hand, for relaxed task independent mechanisms we show next that these are not extendable to $V^{*} \times V_{+, B}$, (in particular, not even to $V_{\epsilon} \times V_{+, B}(!)$ ), except for those that are at the same time affine minimizers or 1-dimensional mechanisms.

\section{B.3.5 $\left.(A, P)\right|_{V_{+} \times V_{+, B}}$ is a (relaxed) task-independent mechanism}

A relaxed task-independent mechanism allocates the two tasks by independent 1-dimensional WMON allocations, up to $\left(s_{1}, s_{2}\right)$ (resp. $\left.\left(t_{1}, t_{2}\right)\right)$ points where the critical value functions $\phi()$ and $\eta()$ of both of these mechanisms have a jump discontinuity. Since there are at most countably many $s_{1}, s_{2}, t_{1}$, and $t_{2}$ where these increasing functions of the 1-dimensional mechanisms can have a jump, it will be easy to handle these $s$ and $t$ values in the following discussion. We assume that the inverse of an increasing function $\phi$ is constant over intervals where the original 
function has a jump, and conversely, the inverse $\phi^{-1}$ has a jump discontinuity in $c$, and $\phi^{-1}(c)$ is defined in an arbitrary monotone way, when the original function is constant $c$ over some interval.

Assume that on $V_{+} \times V_{+, B}$ the payments to the $t$-player, according to the task-independent mechanism are

$$
P_{1}(s)=\phi\left(s_{1}\right), \quad P_{2}(s)=\eta\left(s_{2}\right), \quad P_{12}(s)=\phi\left(s_{1}\right)+\eta\left(s_{2}\right),
$$

where $\phi$ and $\eta$ are arbitrary non-negative increasing functions over $s_{1}, s_{2} \in[0, B)$. We can assume that the values of $\phi$ and $\eta$ are finite, because $R_{\emptyset}=\emptyset$ would hold for the $t$-player in every point $\left(s_{1}, s_{2}\right)$ where $\phi\left(s_{1}\right)=\infty$, or $\eta\left(s_{2}\right)=\infty$.

By Lemma 15, the same payments to the $t$-player hold over $t \in V^{*}$.

In what follows, we consider the allocation and payments to the $s$-player on $V^{*} \times V_{+, B}$ for some carefully chosen $t \in V_{\epsilon} \cap V_{\text {submod }} \subset V^{*}$ values, and will conclude that the mechanism is truthful only if $\Phi \equiv 0$ or $\eta \equiv 0$ (implying a 1-dimensional mechanism), or if $\phi$ and $\eta$ are both linear by the same multiplicative constant, which corresponds to a task-independent affine minimizer 13

We use the notation $\phi(B)=\lim _{s_{1} \rightarrow B^{-}} \phi\left(s_{1}\right)$ and $\eta(B)=\lim _{s_{2} \rightarrow B^{-}} \eta\left(s_{2}\right)$, furthermore $\phi(0)=$ $\lim _{s_{1} \rightarrow 0^{+}} \phi\left(s_{1}\right)$ and $\eta(0)=\lim _{s_{2} \rightarrow 0^{+}} \eta\left(s_{2}\right)$.

The case when $\phi(0)=\phi(B)$, and $\eta(0)=\eta(B)$, yields a constant mechanism. The cases when $\phi(0)=\phi(B)=0$ or $\eta(0)=\eta(B)=0$ correspond to 1-dimensional mechanisms. The case when $\phi(0)=\phi(B)=C>0$ (or $\eta(0)=\eta(B)=C>0)$ on $V_{+} \times V_{+, B}$ is a task-independent mechanism that allocates one of the tasks by a constant mechanism, independently of the bid $s$. We sketch a proof that the latter mechanisms do not extend to $V^{*} \times V_{+, B}$ when $C>0$. Assume the contrary that $\phi \equiv C>0$. Then for the payments

$$
P_{\emptyset}=0, \quad P_{1} \equiv C, \quad P_{2}=\eta\left(s_{2}\right), \quad P_{12}=\eta\left(s_{2}\right)+C
$$

must hold, even on $V^{*} \times V_{+, B}$. This implies that even for every $t \in V^{*}$ in the extension, the allocation is independent of $s_{1}$. That is, for every $t \in V^{*}$ the allocation to the $s$-player either consists of a single region, or has a single horizontal boundary. However, the above payments to the $t$-player imply that if $\phi\left(s_{1}\right) \equiv C>0$ and $\eta\left(s_{2}\right)$ takes on at least two different values, then there exists a $t \in V^{*}$ so that for this fixed $t$, by increasing $s_{2}$, the allocation to the $s$-player gets from $R_{2}^{s}(t)$ into $R_{1}^{s}(t)$ (and this happens even for every $s_{1}$ ). However, the $s$-player cannot have a nonempty $R_{2}^{s}(t)$ and $R_{1}^{s}(t)$ regions at the same time, if it only has horizontal boundaries, a contradiction.

In the rest of this subsection we assume $\phi(0)<\phi(B)$ and $\eta(0)<\eta(B)$.

Definition 22. We will say that

- $t_{1}>0$ is valid, if $t_{1} \in(\phi(0), \phi(B))$, and $\phi^{-1}$ is well-defined (continuous) in the point $t_{1}$;

- $t_{2}>0$ is valid, if $t_{2} \in(\eta(0), \eta(B))$, and $\eta^{-1}$ is well-defined (continuous) in the point $t_{2}$;

- $t=\left(t_{1}, t_{2}, t_{12}\right) \in V$ is valid, if each of $t_{1}, t_{1}^{\prime}=t_{12}-t_{2}, t_{2}$, and $t_{2}^{\prime}=t_{12}-t_{1}$ are valid.

Observe that almost all ${ }^{14} t_{1} \in(\phi(0), \phi(B))$ and almost all $t_{2} \in(\eta(0), \eta(B))$ are valid, because the increasing functions $\phi^{-1}$ and $\eta^{-1}$ have a jump-discontinuity in at most countably many points. Moreover, if $t_{1}$ and $t_{2}$ are valid, then the point $\left(t_{1}, t_{2}, t_{1}+t_{2}\right) \in V_{+}$is valid, and for all $\epsilon^{\prime}<\epsilon$, the points $\left(t_{1}, t_{2}, t_{1}+t_{2}-\epsilon^{\prime}\right)$ are in $V_{\epsilon} \cap V_{\text {submod }}$, and they are valid for almost all $\epsilon^{\prime}$. It follows that the set of all valid $t$ points is dense in $V_{\epsilon} \cap V_{\text {submod }}$, at least if we restrict

\footnotetext{
${ }^{13} \mathrm{~A}$ task-independent affine minimizer minimizes among $t_{12}, t_{1}+\mu s_{2}+\gamma_{1}, \mu s_{1}+t_{2}+\gamma_{2}, \mu\left(s_{1}+s_{2}\right)+\gamma_{1}+\gamma_{2}$ for some given constants $\mu, \gamma_{1}, \gamma_{2}$.

${ }^{14}$ meaning: 'except for a countable subset'
} 
attention to $t_{1} \in\left(\phi(0)+\epsilon^{\prime}, \phi(B)-\epsilon^{\prime}\right)$. Our goal is to show that the restriction of $\phi^{-1}$ to the valid points of $\left(\phi(0)+\epsilon^{\prime}, \phi(B)-\epsilon^{\prime}\right)$ is identical to a linear function, and analogously for $t_{2}$ and $\eta^{-1}$; moreover $\phi^{-1}$ and $\eta^{-1}$ have the same slope. Thus, since a restriction of each of $\phi$ and $\eta$ to a dense subset is linear, and they are increasing, in fact, they cannot have jump discontinuities at all, and are (allover) linear functions with the same slope. Finally, by taking $\epsilon^{\prime} \rightarrow 0$, we obtain linearity over $(\phi(0), \phi(B))$, and $(\eta(0), \eta(B))$ respectively. This will prove that $\left.A\right|_{V_{+} \times V_{+, B}}$ is a task-independent affine minimizer, and $A$ is necessarily the same affine minimizer on $V^{*} \times V_{+, B}$.

First we argue that for all valid $t$ the regions $R_{\emptyset}^{s}(t) \subset V_{+}$and $R_{1}^{s}(t) \subset V_{+}$are nonempty, in particular there exist $s \in V_{+, B}$ on the boundary of these two regions. Since $R_{\emptyset}^{s}(t)$ is nonempty, for valid $t$ the virtual payments $P_{\alpha}^{s}(t)$ of the $s$-player are well-defined. Further, an $s$ on the boundary will help us to obtain a formula for $P_{1}^{s}(t)$.

Consider an arbitrary fixed valid $t \in V$. Since $t_{2}<\eta(B)$, there exist $s \in V_{+, B}$ so that $t_{2}<\eta\left(s_{2}\right)$, or equivalently $\eta^{-1}\left(t_{2}\right)<s_{2}$. For every such $s$ and the fixed $t$, the $t$-player receives task 2. Moreover, since $\eta^{-1}$ is continuous in $t_{2}$, the same holds (there exist proper $s_{2}$ values globally) for all points in some neighborhood of $t$. Now, for every such $s$, the $s$ is on the boundary of $R_{\emptyset}^{s}$ and $R_{1}^{s}$, iff our fixed point $t$ is on the boundary of $R_{12}(s)$ and $R_{2}(s){ }^{15}$ On the other hand, $s$ is on the boundary of $R_{\emptyset}^{s}$ and $R_{1}^{s}$, iff $s_{1}=P_{1}^{s}$ (here we use that $s$ can not get task 2), and $t$ is on the boundary of $R_{12}(s)$ and $R_{2}(s)$ iff $t_{12}-t_{2}=P_{12}(s)-P_{2}(s)=\phi\left(s_{1}\right)$. Observe that such an $s_{1}$ does exist, given that $t_{12}-t_{2}$ is valid, i.e. is within the range of valid $t_{1}$ values and is a continuity point of $\phi^{-1}$. In summary, for the fixed valid $t$, and the found $s$ it must hold that

$$
P_{1}^{s}(t)=s_{1}=\phi^{-1}\left(t_{12}-t_{2}\right),
$$

which, for any valid $t$ yields the formula for $P_{1}^{s}(t)$ independently of $s$. Analogously, we obtain for valid $t$ that

$$
P_{2}^{s}(t)=\eta^{-1}\left(t_{12}-t_{1}\right)
$$

Next we determine $P_{12}^{s}$ in two different ways for every valid $t$. First of all we claim that if $t$ is valid then $R_{12}^{s}(t)$ is nonempty. Indeed, by the validity of $t$, there exist $s$ such that $s_{1}<\phi^{-1}\left(t_{1}\right)$, and $s_{2}<\eta^{-1}\left(t_{2}\right)$, and $s_{2}<\eta^{-1}\left(t_{12}-t_{1}\right)$. For all such $s$ it holds that $t_{1}>\phi\left(s_{1}\right)=P_{1}(s)$, and $t_{2}>\eta\left(s_{2}\right)=P_{2}(s)$, and $t_{12}>\eta\left(s_{2}\right)+t_{1}>\eta\left(s_{2}\right)+\phi\left(s_{1}\right)=P_{12}(s)$. Therefore $t \in R_{\emptyset}(s)$. This implies that the (open) set of all such $s$ must belong to $R_{12}(t)$.

Second, we claim that there exists an $s^{\prime}$ on the boundary of $R_{12}^{s}(t)$ and $R_{2}^{s}(t)$, and another $s^{\prime \prime}$ on the boundary of $R_{12}^{s}(t)$ and $R_{1}^{s}(t)$. Indeed, let $s^{\prime}$ be such that $s_{1}^{\prime}=\phi^{-1}\left(t_{1}\right)$, and $s_{2}^{\prime}<\eta^{-1}\left(t_{2}\right)$, and $s_{2}^{\prime}<\eta^{-1}\left(t_{12}-t_{1}\right)$. Then, $t_{2}>\eta\left(s_{2}^{\prime}\right)=P_{2}\left(s^{\prime}\right)$, and $t_{12}>\eta\left(s_{2}^{\prime}\right)+t_{1}=\eta\left(s_{2}^{\prime}\right)+\phi\left(s_{1}^{\prime}\right)=P_{12}\left(s^{\prime}\right)$, so the $t$-player does not get task 2 even with a bid in a small enough neighborhood of $t$, and because of $t_{1}=\phi\left(s_{1}^{\prime}\right)$ it must be on the boundary of $R_{1}\left(s^{\prime}\right)$ and $R_{\emptyset}\left(s^{\prime}\right)$, so $s^{\prime}$ is on the boundary of $R_{2}^{s}(t)$ and $R_{12}^{s}(t)$ (here we use that $s_{1}^{\prime}$ is uniquely defined as $\phi^{-1}\left(t_{1}\right)$ ). The proof is analogous for $s^{\prime \prime}$.

By the position of $s^{\prime}$,

$$
P_{12}^{s}-P_{2}^{s}=s_{1}^{\prime}=\phi^{-1}\left(t_{1}\right)
$$

the second equality follows from the fact that for $s^{\prime}$ the $t$ must be on the boundary of $R_{\emptyset}$ and $R_{1}$. Plugging in $P_{2}^{s}$ we obtain (using also that $\phi^{-1}$ and $\eta^{-1}$ are well-defined in $t$ )

$$
P_{12}^{s}=\phi^{-1}\left(t_{1}\right)+\eta^{-1}\left(t_{12}-t_{1}\right) .
$$

On the other hand, using $s^{\prime \prime}$ we obtain by analogous argument that

$$
P_{12}^{s}=\eta^{-1}\left(t_{2}\right)+\phi^{-1}\left(t_{12}-t_{2}\right) .
$$

This yields that

$$
\phi^{-1}\left(t_{1}\right)+\eta^{-1}\left(t_{12}-t_{1}\right)=\eta^{-1}\left(t_{2}\right)+\phi^{-1}\left(t_{12}-t_{2}\right)
$$

\footnotetext{
${ }^{15}$ In fact, the boundary of the $t$-player is allowed to have a jump in $s_{1}=\phi^{-1}\left(t_{1}\right)$.
} 
for every valid $t$.

Let $\epsilon^{\prime}<\epsilon$. Assume for a moment that all $t \in V_{\epsilon} \cap V_{\text {submod }}$ with $t_{1} \in\left(\phi(0)+\epsilon^{\prime}, \phi(B)-\epsilon^{\prime}\right)$ and $t_{2} \in\left(\eta(0)+\epsilon^{\prime}, \eta(B)-\epsilon^{\prime}\right)$ are valid, and consider such a $t$. Increasing now only $t_{1}$ by an arbitrary positive $\delta<\epsilon^{\prime}$, the right side of the last equality does not change. This is possible only if $\phi^{-1}\left(t_{1}\right)$ and $\eta^{-1}\left(t_{12}-t_{1}\right)$ increases, resp. decreases by the same value $\Delta:=\phi^{-1}\left(t_{1}+\delta\right)-\phi^{-1}\left(t_{1}\right)=$ $\eta^{-1}\left(t_{12}-t_{1}\right)-\eta^{-1}\left(t_{12}-t_{1}-\delta\right)$.

Now fix $t_{1}, t_{2}$ and $\delta$ (thereby fixing $\Delta$ ), and consider every $t_{12} \in\left(t_{1}+t_{2}-\epsilon^{\prime}, t_{1}+t_{2}\right)$. For every such $t_{12}$ holds by the same argument $\eta^{-1}\left(t_{12}-t_{1}\right)-\eta^{-1}\left(t_{12}-t_{1}-\delta\right)=\Delta$ with the fixed $\Delta$. The last statement can be rewritten as $\eta^{-1}(x)-\eta^{-1}(x-\delta)=\Delta$ for every $x \in\left(t_{2}-\epsilon^{\prime}, t_{2}\right)$. Now taking $\delta / 2^{r}$ instead of $\delta$ and letting $r \rightarrow \infty$, by the monotonicity of $\eta^{-1}$ this implies that $\eta^{-1}$ must be linear on the interval $\left(t_{2}-\epsilon^{\prime}, t_{2}\right)$. (The proof of this is analogous to the proof of Lemma 11.)

As a final step, change $t_{2}$ to an arbitrary other valid $t_{2}^{\prime}$. Here again, by the same argument $\eta^{-1}$ is linear over $\left(t_{2}^{\prime}-\epsilon^{\prime}, t_{2}^{\prime}\right)$. Since such overlapping open intervals cover the interval $(\eta(0)+$ $\left.\epsilon^{\prime}, \eta(B)-\epsilon^{\prime}\right)$, the function $\eta^{-1}$ must be a single linear function over $\left(\eta(0)+\epsilon^{\prime}, \eta(B)-\epsilon^{\prime}\right)$. By taking the limit $\epsilon^{\prime} \rightarrow 0$ we obtain that $\phi^{-1}$ is linear over $(\eta(0), \eta(B))$. By symmetric argument, $\phi^{-1}$ is linear, too, and $\phi^{-1}$ and $\eta^{-1}$ have the same multiplicative constant $\Delta / \delta$ as follows from $\Delta=\phi^{-1}\left(t_{1}+\delta\right)-\phi^{-1}\left(t_{1}\right)=\eta^{-1}\left(t_{12}-t_{1}\right)-\eta^{-1}\left(t_{12}-t_{1}-\delta\right)$.

Now, how do we go around non-valid points $t \in V_{\epsilon} \cap V_{\text {submod }}$ ? By counting arguments it can be shown that the grid of rational $\left(t_{1}, t_{2}\right)$ pairs can be translated by a real vector, so that all translated pairs are valid. After this, a real $\delta$ can be found so that every corresponding $\left(t_{1}, t_{2}, t_{1}+t_{2}-\delta \cdot k / 2^{r}\right)$ is valid, too. Using only these numbers we obtain that $\phi$ and $\eta$ are linear on the translated rational grid, so given that they are monotone increasing, they must be linear functions allover. This concludes the proof that the allocation function is a task-independent affine minimizer on $V^{*} \times V_{+, B}$.

\section{B.3.6 Mechanisms with high approximation ratio}

We proved above that any truthful mechanism $(A, P)$ on $V^{*} \times V_{+, B}$ is (1) relaxed affine minimizer, (2) a one-dimensional mechanism, or (3) a constant mechanism. It remains to prove that in cases (2), and (3), the approximation ratio is high. This will conclude the proof of Theorem 5 .

Lemma 16. If $(A, P)$ on $V^{*} \times V_{+, B}$ is a one-dimensional mechanism, or a constant mechanism, there exists an instance for which the makespan of $A$ is at least $\sqrt{B}$ and the approximation ratio is at least $\sqrt{B}$.

Proof. Assume first that $A$ is a constant mechanism. By the assumption that $R_{\emptyset}(s) \neq \emptyset$ for every $s$, it must be the case that the constant mechanism is independent of $s$.

If, for $t=(\sqrt{B}, \sqrt{B})$ at least one of the tasks is given to the $t$-player, then the makespan is $2 \sqrt{B}$ and the approximation ratio is unbounded, because $s$ can be chosen arbitrarily small. If for $t=(\sqrt{B}, \sqrt{B})$ both tasks are given to the $s$-player, then we can choose $s=(B, B)$ to prove approximation ratio of at least $\sqrt{B}$.

Assume now that $A$ is a bundling allocation, and consider the input $\left(t_{1}=\sqrt{B}, t_{2}=0, s_{1}=\right.$ $\left.0, s_{2}=\sqrt{B}\right)$. Either both tasks are given to the $t$-player, or both are given to the $s$-player, so the makespan is $\sqrt{B}$ and the approximation ratio is unbounded.

Finally, assume w.l.o.g., that $A$ realizes only the two allocations $\alpha_{t}=\emptyset$, or $\alpha_{t}=1$. Then set $s_{2}=\sqrt{B}$, and $t_{1}=t_{2}=0$. Since the $s$-player must receive task 2 , the approximation is again unbounded. 